The Astrophysical Journal, 684:212-225, 2008 September 1

(C) 2008. The American Astronomical Society. All rights reserved. Printed in U.S.A.

\title{
GRAVITATIONAL INSTABILITY IN RADIATION PRESSURE-DOMINATED BACKGROUNDS
}

\author{
TodD A. Thompson \\ Department of Astronomy and Center for Cosmology and Astroparticle Physics, The Ohio State University, Columbus, OH 43210; \\ thompson@astronomy.ohio-state.edu \\ Received 2007 November 21; accepted 2008 April 11
}

\begin{abstract}
I consider the physics of gravitational instabilities in the presence of dynamically important radiation pressure and gray radiative diffusion. For any nonzero radiation diffusion rate on an optically thick scale, the medium is unstable unless the classical gas-only isothermal Jeans criterion is satisfied. When diffusion is "slow," even though the Jeans instability is stabilized by radiation pressure on scales smaller than the adiabatic Jeans length, on these same spatial scales the medium is unstable to a diffusive mode. In this regime, neglecting gas pressure, the characteristic growth timescale is independent of spatial scale and given by $\left(3 \kappa c_{s}^{2}\right) /(4 \pi G c)$, where $c_{s}$ is the adiabatic sound speed. This timescale is that required for a fluid parcel to radiate away its thermal energy content at the Eddington limit, the Kelvin-Helmholz timescale for a radiation pressure-supported self-gravitating object. In the limit of "rapid" diffusion, radiation does nothing to suppress the Jeans instability and the medium is dynamically unstable unless the gas-only Jeans criterion is satisfied. I connect with treatments of Silk damping in the early universe. I discuss several applications, including photons diffusing in regions of extreme star formation (starburst galaxies and parsec-scale AGN disks), and the diffusion of cosmic rays in normal galaxies and galaxy clusters. The former (particularly, starbursts) are "rapidly" diffusing and thus cannot be supported against dynamical instability in the linear regime by radiation pressure alone. The latter are more nearly "slowly" diffusing. I speculate that the turbulence in starbursts may be driven by the dynamical coupling between the radiation field and the self-gravitating gas, perhaps mediated by magnetic fields, and that this diffusive instability operates in individual massive stars.
\end{abstract}

Subject headings: galaxies: starburst

\section{INTRODUCTION}

Standard treatments of the Jeans instability assume the medium is homogeneous and isotropic and governed by a barotropic equation of state. Employing the "Jeans swindle" so that the Poisson equation is satisfied in an ad hoc way with no background gradients in density, the dispersion relation

$$
\omega^{2}=c_{g}^{2} k^{2}-4 \pi G \rho
$$

follows from a linear analysis. Here, $c_{g}$ is the gas sound speed and $\rho$ is the mass density. The Jeans instability is long wavelength; for scales larger than the Jeans length,

$$
2 \pi k_{\mathrm{J}}^{-1}=\lambda_{\mathrm{J}}=c_{g}(\pi / G \rho)^{1 / 2},
$$

the system is dynamically unstable under the action of a perturbation to the density and the attending increase in the gravitational potential (Jeans 1928; e.g., Binney \& Tremaine 1987). On scales smaller than $\lambda_{\mathrm{J}}$ the medium responds to a compression with a restoring pressure force. Equation (2) can be obtained by equating the acoustic sound crossing timescale on a scale $\lambda_{\mathrm{J}}$ with the dynamical timescale. Equivalently, equation (2) may be read as expressing the fact that for stability, the total thermal energy of the medium within a volume $\lambda_{\mathrm{J}}^{3}$ must exceed the gravitational potential energy. When the system is unstable, it continuously and spontaneously transitions to states of lower total energy by liberating thermal energy (e.g., Chandrasekhar 1961).

The purpose of this paper is to understand how the classical gas Jeans criterion is modified by radiation and to ask in which astrophysical environments such a modification might be important. Although there are many treatments in the literature of both the Jeans instability (e.g., Jeans 1928; Ledoux 1951; Chandrasekhar
1954, 1961; Mestel 1965; Lynden-Bell 1966) and the physics of radiating flows (e.g., Mihalas \& Mihalas 1983, 1984, and references therein; Spiegel 1957; Field 1971; Kaneko et al. 1976, 2000; Bisnovatyi- Kogan \& Blinnikov 1978, 1979; Dzhalilov et al. 1992; Zhugzhda et al. 1993; Arons 1992; Bogdan et al. 1996; Gammie 1998; Blaes \& Socrates 2001, 2003; Socrates et al. 2005), there has been relatively little work on self-gravitating environments where radiation might play an important dynamical role (however, see Kaneko \& Morita 2006; Vranjes \& Cadez 1990; Vranjes 1990).

Perhaps the first and most familiar treatment of self-gravitating radiation pressure-dominated media was carried out by Silk (1967, 1968), and then extended by Peebles \& Yu (1970) and Weinberg (1971), in the context of acoustic wave damping of primeval fluctuations by radiative diffusion- "Silk damping" (see also Hu \& Sugiyama 1996; Dodelson 2003). However, these works focus specifically on the damping rate of acoustic fluctuations and the generation of entropy and did not delineate how the Jeans criterion is modified on scales larger than the gas-only Jeans length when radiation is dynamically dominant and diffusing. They also do not discuss the physics of slow nondynamical diffusive modes.

This paper is motivated by astrophysical systems where selfgravity and radiation are essential. These include sites of extreme massive star formation such as compact starburst galaxies and the parsec-scale disks or obscuring "tori" thought to attend the process of fueling active galactic nuclei. These environments are marked by high radiation energy density and high gas density, as well as optical depths to their own dust-reprocessed infrared radiation that may significantly exceed unity $(\S 3$ and, e.g., Pier \& Krolik 1992; Goodman 2003; Sirko \& Goodman 2003; Thompson et al. 2005, hereafter TQM05; Chang et al. 2007). In each of these systems radiation pressure can be comparable to gravity, and the associated photon energy density is rivaled only by the energy density in turbulence and, potentially, the contributions from 
cosmic rays and magnetic fields. ${ }^{1}$ The very high radiation energy densities in these systems led TQM05 to propose a theory of marginally Toomre-stable radiation pressure supported starburst and AGN disks (see also Scoville et al. 2001; Scoville 2003). In addition, this analysis may be of some interest for the stability of individual massive stars and for self-gravitating media whose pressure is dominated by cosmic rays, either in normal star-forming galaxies or galaxy clusters.

In $\S 2$, I present a simple linear analysis of the gravitational instability. Appendix A discusses uniform rotation in the background medium. More detailed treatments of radiation transport are considered in Appendix B (see also Kaneko \& Morita 2006). In $\S 3$, I discuss the relevance of the results derived for a number of astrophysical environments and $\S 4$ provides a summary.

\section{GRAVITATIONAL INSTABILITY WITH RADIATION}

Here, I describe the simplest nontrivial treatment of the Jeans problem with radiation pressure and diffusion that captures the physics needed for a qualitative understanding (see Appendix B for a more detailed treatment). The equations express continuity, momentum and total energy conservation, self-gravity, and equilibrium optically thick radiative diffusion (e.g., Mihalas \& Mihalas 1984). They are

$$
\begin{gathered}
\frac{\partial \rho}{\partial t}+\nabla \cdot(\rho \boldsymbol{v})=0 \\
\frac{\partial \boldsymbol{v}}{\partial t}+\boldsymbol{v} \cdot \nabla \boldsymbol{v}=-\frac{1}{\rho} \nabla P-\nabla \Phi \\
\frac{\partial U}{\partial t}+\boldsymbol{v} \cdot \nabla U+(U+P) \nabla \cdot \boldsymbol{v}=-\nabla \cdot \boldsymbol{F}, \\
\nabla^{2} \Phi=4 \pi G \rho \\
\boldsymbol{F}=-\frac{c}{3 \kappa \rho} \nabla u_{r} .
\end{gathered}
$$

Here $U=u_{g}+u_{r}$ and $P=p_{g}+p_{r}$ are the total internal energy density and pressure, and the subscripts $r$ and $g$ refer to the radiation and the gas, respectively. The radiation pressure force $\kappa \boldsymbol{F} / c$ is contained in the $\nabla P$ term in equation (4). In addition, $p_{g}=$ $\rho k_{\mathrm{B}} T / m_{p}, u_{r}=a T^{4}=3 p_{r}, u_{g}=p_{g} /(\gamma-1), \rho$ is the gas mass density, $\gamma$ is the adiabatic index of the gas, $\boldsymbol{F}$ is the radiative flux, and $\kappa$ is the opacity.

For simplicity, I take $\kappa$ constant and I do not distinguish between the Planck-, flux-, and Rosseland-mean opacities. The above equations also neglect the time dependence of the radiation field, and they assume that the radiation and gas temperatures are exactly equal (see Appendix B; see also Mihalas \& Mihalas 1984; Gammie 1998; Blaes \& Socrates 2003; Kaneko \& Morita 2006). Because the Eddington approximation has been made, the effects of photon viscosity have been neglected (e.g., Weinberg 1971; Agol \& Krolik 1998). In addition, in considering extreme star formation environments where radiation is reprocessed by dust, the above equations neglect the two-fluid nature of the coupled dust-gas system; that is, they assume perfect collisional and energetic coupling between the dust and gas (see $\S 3$ ). Finally, no terms representing sources of optically thin radiative heating or cooling are included.

I consider perturbations of the form $q \rightarrow q+\delta q \exp (i \boldsymbol{k} \cdot \boldsymbol{x}-$ $i \omega t)$, keep only linear terms, employ the Jeans swindle, and take

\footnotetext{
${ }^{1}$ For a recent assessment of the strength of magnetic fields in starburst galaxies, see Thompson et al. (2006). For a discussion of cosmic-ray feedback in galaxies, see Socrates et al. (2008).
}

the medium and radiation field as homogeneous, isotropic, and in radiative equilibrium: $\rho=$ const., $P=$ const., $U=$ const., and $\boldsymbol{v}=\nabla \cdot \boldsymbol{F}=\boldsymbol{F}=0$. The perturbed equations are

$$
\begin{gathered}
-i \omega \delta \rho+i \rho \boldsymbol{k} \cdot \delta \boldsymbol{v}=0, \\
-i \omega \delta \boldsymbol{v}+i \boldsymbol{k}(\delta P / \rho)+i \boldsymbol{k} \delta \Phi=0, \\
-i \omega \delta U+(U+P) i \boldsymbol{k} \cdot \delta \boldsymbol{v}+i \boldsymbol{k} \cdot \delta \boldsymbol{F}=0, \\
-k^{2} \delta \Phi-4 \pi G \delta \rho=0, \\
i \boldsymbol{k} \delta u_{r}+(3 \kappa \rho / c) \delta \boldsymbol{F}=0 .
\end{gathered}
$$

The thermodynamic perturbations to the total pressure and energy density are

$$
\begin{gathered}
\delta P=\left.\frac{\partial P}{\partial \rho}\right|_{T} \delta \rho+\left.\frac{\partial P}{\partial T}\right|_{\rho} \delta T \\
\delta U=\left.\frac{\partial U}{\partial \rho}\right|_{T} \delta \rho+\left.\frac{\partial U}{\partial T}\right|_{\rho} \delta T .
\end{gathered}
$$

Note that $\left.\left(\partial p_{r} / \partial \rho\right)\right|_{T}=\left.\left(\partial u_{r} / \partial \rho\right)\right|_{T}=0$ so that $\left.(\partial P / \partial \rho)\right|_{T}=$ $\left.\left(\partial p_{g} / \partial \rho\right)\right|_{T}=c_{T}^{2}$ - that is, only the gas makes a contribution to the total isothermal sound speed, $\left(\partial P /\left.\partial \rho\right|_{T}\right)^{1 / 2}=c_{T}$, in equation (13). The perturbation to the radiation energy density is written as

$$
\delta u_{r}=4 a T^{3} \delta T=A \delta T,
$$

where the last equality defines $A=\partial u_{r} / \partial T$. Combining these thermodynamic relations with the perturbation equations, one finds that

$$
0=\left(\omega^{2}-c_{T}^{2} k^{2}+4 \pi G \rho\right)\left(1+\frac{i \tilde{\omega}}{\omega}\right)+\left(c_{T}^{2}-c_{s}^{2}\right) k^{2}
$$

where

$$
\tilde{\omega}=\frac{c k^{2}}{3 \kappa \rho}\left(\frac{A}{C_{V}}\right)
$$

is the diffusion rate on a scale $k^{-1}, C_{V}=\left.(\partial U / \partial T)\right|_{\rho}$ is the total specific heat, $c_{s}^{2}=\left.(\partial P / \partial \rho)\right|_{s}$ is the square of the adiabatic sound speed for the gas and radiation, $S$ is the total entropy, and the identity

$$
\left.\frac{\partial P}{\partial \rho}\right|_{S}=\left.\frac{\partial P}{\partial \rho}\right|_{T}+\left.\frac{\partial P}{\partial U}\right|_{\rho}\left[\left(\frac{U+P}{\rho}\right)-\left.\frac{\partial U}{\partial \rho}\right|_{T}\right]
$$

has been employed. Expanding the dispersion relation and combining terms, equation (16) becomes

$\omega^{3}+i \omega^{2} \tilde{\omega}-\omega\left(c_{s}^{2} k^{2}-4 \pi G \rho\right)-i \tilde{\omega}\left(c_{T}^{2} k^{2}-4 \pi G \rho\right)=0$.

Note that the dimensionless ratio

$$
\frac{A}{C_{V}}=\frac{\partial u_{r}}{\partial T}\left(\left.\frac{\partial U}{\partial T}\right|_{\rho}\right)^{-1}=\left(1+\frac{u_{g}}{4 u_{r}}\right)^{-1}
$$

that appears in equation (17) for $\tilde{\omega}$ approaches unity in the limit $u_{g} /\left(4 u_{r}\right) \rightarrow 0$, and zero in the limit $u_{g} /\left(4 u_{r}\right) \rightarrow \infty$. Therefore, as 
$u_{r} / u_{g} \rightarrow 0, c_{s}^{2} \rightarrow c_{s, g}^{2}=\gamma c_{T}^{2}$, and $\left(A / C_{V}\right) \rightarrow 0$, equation (19) reduces to

$$
\omega^{3}-\omega\left(\gamma c_{T}^{2} k^{2}-4 \pi G \rho\right)=0
$$

in the gas pressure-dominated limit, fully analogous to the classical Jeans criterion in equation (1), but includes the entropy mode $\omega=0$ (e.g., Lithwick \& Goldreich 2001) and explicitly contains the adiabatic gas sound speed $c_{s, g}^{2}=\gamma c_{T}^{2}$. In the opposite, radiation pressure-dominated limit, $u_{r} / u_{g} \rightarrow \infty, c_{s}^{2} \rightarrow c_{s, r}^{2}=$ $\left(4 p_{r} / 3 \rho\right) \gg c_{T}^{2}$. Neglecting gravity, the dispersion relation for radiation pressure acoustic waves under the assumption of optically thick equilibrium radiative diffusion, $\omega\left(\omega^{2}+i \tilde{\omega} \omega-c_{s, r}^{2} k^{2}\right) \approx 0$, is obtained from equation (19).

\subsection{Dimensionless Numbers}

Three dimensionless numbers determine the character of the modes admitted by equation (19). The first measures the importance of gas pressure alone in supporting the medium on a scale $k^{-1}$ :

$$
\vartheta_{T}=c_{T}^{2} k^{2} /(4 \pi G \rho)
$$

The criterion $\vartheta_{T}>1$ is the classical gas-only Jeans criterion for gravitational stability (cf. eq. [2]); $\vartheta_{T}$ is the "isothermal Jeans number." The isothermal Jeans length follows by taking $\vartheta_{T}=1$ :

$$
\lambda_{\mathrm{J}, T}=2 \pi / k_{\mathrm{J}, T}=2 \pi c_{T} /(4 \pi G \rho)^{1 / 2} .
$$

The second and third dimensionless ratios combine to determine the importance of radiation pressure. The first is

$$
\vartheta_{s}=c_{s}^{2} k^{2} /(4 \pi G \rho)
$$

the "adiabatic Jeans number," analogous to $\vartheta_{T}$, but which includes the contribution from radiation pressure. Taking $\vartheta_{s}=1$ defines the adiabatic Jeans length:

$$
\lambda_{\mathrm{J}, s}=2 \pi / k_{\mathrm{J}, s}=2 \pi c_{s} /(4 \pi G \rho)^{1 / 2} .
$$

The second ratio is

$$
\chi=\frac{c k^{2}}{3 \kappa \rho}\left(\frac{A}{C_{V}}\right) \frac{1}{(4 \pi G \rho)^{1 / 2}},
$$

a measure of the diffusion rate. The limits of rapid $(\chi \gg 1)$ and slow $(\chi \ll 1)$ diffusion are considered in $\S \S 2.2$ and 2.3, respectively.

In analogy with the classical Jeans criterion, one might guess that if $\vartheta_{s}$ is larger than unity, then in the limit of slow diffusion the medium is stable. This turns out to be false, as I show in $\S 2.3$. In fact, if $\vartheta_{T}<1$ on a scale $k^{-1}$, then the medium is unstable regardless of $\vartheta_{s}$.

Using $\xi^{2}=\omega^{2} /(4 \pi G \rho)$, and the definitions for $\vartheta_{T}, \vartheta_{s}$, and $\chi$, equation (19) can be written as

$$
\xi^{3}+i \chi \xi^{2}-\xi\left(\vartheta_{s}-1\right)-i \chi\left(\vartheta_{T}-1\right)=0 .
$$

\subsection{Rapid Diffusion}

In the limit of rapid diffusion ( $\chi \gg \vartheta_{s}, \vartheta_{T}$, and 1), the three roots of equation (27) are

$$
\begin{gathered}
\xi \approx \pm\left(\vartheta_{T}-1\right)^{1 / 2}-i \frac{\left(\vartheta_{s}-\vartheta_{T}\right)}{2 \chi}, \\
\xi \approx-i \chi+i \frac{\left(\vartheta_{s}-\vartheta_{T}\right)}{\chi},
\end{gathered}
$$

to first order in $\chi^{-1}$. When $\vartheta_{T}>1$, the roots in equation (28) correspond to stable radiation- and gravity-modified gas acoustic waves. For large $\vartheta_{T}$, these modes propagate at the isothermal sound speed of the gas; large $\chi$ ensures isothermality. In the limit $\vartheta_{T} \rightarrow 0$ and $\chi \gg \vartheta_{s}$, equation (28) is simply $\xi \approx \pm i$ and the medium is dynamically unstable. This is the classical gasonly isothermal Jeans instability. Note that the limit of rapid diffusion in equation (28) is distinct from the high- $k$ limit, because at high- $k$ gravity, which dictates stability/instability, disappears. However, to make an apposite comparison with the literature I take the high- $k$ limit and for the acoustic modes I find that

$$
\omega \approx \pm c_{T} k-i \frac{2 \kappa}{3 c} u_{r}\left(1+\frac{3 p_{g}}{4 u_{r}}\right)^{2},
$$

in agreement with Blaes \& Socrates (2003, their eq. [62]). Equation (29) corresponds to the purely damped radiation diffusion wave.

\subsection{Slow Diffusion}

In the limit of slow diffusion $\left(\chi \ll \vartheta_{s}, \vartheta_{T}\right.$, and 1$)$,

$$
\begin{gathered}
\xi \approx \pm\left(\vartheta_{s}-1\right)^{1 / 2}-\frac{i \chi}{2}\left(\frac{\vartheta_{s}-\vartheta_{T}}{\vartheta_{s}-1}\right), \\
\xi \approx-i \chi\left(\frac{\vartheta_{T}-1}{\vartheta_{s}-1}\right) .
\end{gathered}
$$

If $\vartheta_{s}>1$, equation (31) corresponds to two stable damped gravity-modified radiation acoustic waves. ${ }^{2}$ For $\vartheta_{s} \gg 1$ and $\vartheta_{s} \gg \vartheta_{T}$, the damping rate for these radiation acoustic waves is simply $\chi / 2$. Conversely, when $\vartheta_{s}<1$ (and, thus, $\vartheta_{T}<1$ ) - that is, on scales larger than the adiabatic Jeans length (eq. [25])the medium is dynamically unstable to the Jeans instability: $\xi \rightarrow \pm i$.

Equation (32) is key. It says that there is an intermediate range in spatial scale $k^{-1}$, larger than isothermal Jeans length (eq. [23]) and smaller than the adiabatic Jeans length (eq. [25]), that is always unstable. For negligible gas pressure $\left(\vartheta_{T} \rightarrow 0\right)$, it is precisely when the adiabatic Jeans number $\vartheta_{s}$ is greater than unity and the dynamical Jeans instability is suppressed in equation (31) that the diffusive mode in equation (32) is unstable. Even for arbitrarily large $\vartheta_{s}$ and small $\chi$, if the classical gas-only Jeans criterion indicates instability - that is, if $\vartheta_{T}<1$ - then the medium is unstable.

In a highly radiation pressure-dominated medium with $\vartheta_{T} \ll$ $1 \ll \vartheta_{s}$, this diffusive mode grows at a rate

$$
\omega \approx i \frac{4 \pi G}{3 \kappa} \frac{c}{c_{s, r}^{2}}
$$

in the high- $k$ limit, independent of spatial scale. This expression is easy to understand as the rate at which a self-gravitating fluid parcel of volume $V$ and mass $M$ radiates its total thermal energy content $\left[e \sim(4 / 3) u_{r} V\right]$ at the Eddington limit $(\dot{e}=$ $4 \pi G M c / \kappa)$; it is the inverse of the Kelvin-Helmholz timescale: $t_{\mathrm{KH}}^{-1} \sim \dot{e} / e \sim 4 \pi G c /\left(3 \kappa c_{s, r}^{2}\right)$.

Equivalently, the only terms from the Euler and energy equations that contribute to this branch of the dispersion relation are

\footnotetext{
2 Note that the real part of $\xi$ is modified by $\chi$ at the level $\xi \approx \pm\left(\vartheta_{s}-\right.$ $1)^{1 / 2} \mp\left(\chi^{2} / 2\right)\left(\vartheta_{s}-\vartheta_{T}\right)\left(\vartheta_{s}-1\right)^{-3 / 2}$ in eq. (31) if the second-order term in $\chi$ is
kept.
} 
the approximate equalities $\boldsymbol{k} \delta P / \rho \approx-\boldsymbol{k} \delta \Phi$ and $(U+P) \boldsymbol{k} \cdot \delta \boldsymbol{v} \approx$ $-\boldsymbol{k} \cdot \delta \boldsymbol{F}$ (cf. eqs. [9] and [10]). Combining the energy equation with the continuity equation, $(\delta \rho / \rho)=(\boldsymbol{k} \cdot \delta \boldsymbol{v}) / \omega$, and assuming that the medium is radiation pressure dominated,

$$
\omega=i\left(\frac{c k^{2}}{\kappa \rho}\right)\left(\frac{\delta p_{r}}{\delta \rho}\right)\left(\frac{1}{4} \frac{\rho}{p_{r}}\right) .
$$

On the other hand, the approximate equality $\boldsymbol{k} \delta P / \rho \approx-\boldsymbol{k} \delta \Phi$ implies that

$$
\frac{\delta p_{r}}{\delta \rho}=\frac{4 \pi G \rho}{k^{2}}
$$

Combining equations (34) and (35), one finds precisely equation (33). Substituting into the continuity equation, I find that

$$
\boldsymbol{k} \cdot \delta \boldsymbol{v}=i \frac{c k^{2}}{\kappa \rho} \frac{\delta p_{r}}{4 p_{r}}=i\left(\frac{4 \pi G \delta \rho}{\kappa \rho c}\right)\left(\frac{\rho c^{2}}{4 p_{r}}\right)
$$

which relates the density and velocity perturbations.

Therefore, in a radiation pressure-dominated medium with slow diffusion (see $\S 2.5$ ) the characteristic time for collapse on scales smaller than the adiabatic Jeans length is $t_{\mathrm{KH}}$, not the dynamical timescale. Although the growth timescale becomes long as $\kappa$ and $c_{s, r}$ become large, for $\chi \neq 0$ the medium is never formally stable if $\vartheta_{T}<1$. In addition, the existence of this instability does not require a pure radiation-only gas with adiabatic index of $4 / 3$. Written another way, the growth timescale for this diffusive instability at large $k$ is

$$
t_{\mathrm{KH}} \approx t_{\mathrm{diff}}\left(t_{\mathrm{dyn}} / t_{r}\right)^{2}
$$

in an optically thick, slowly diffusing, radiation pressuredominated medium, where $t_{r}=\left(c_{s, r} k\right)^{-1}$ is the radiation pressure acoustic sound crossing timescale, and $t_{\mathrm{diff}}=3 \kappa \rho / \mathrm{ck}^{2}$ and $t_{\mathrm{dyn}}=(4 \pi G \rho)^{-1 / 2}$ are the diffusion and dynamical timescales, respectively.

\subsection{Criterion for Existence of the Radiation Acoustic Mode}

If the adiabatic Jeans number is larger than unity $\left(\vartheta_{s}>1\right)$, then when the radiation acoustic sound crossing timescale on a scale $k^{-1}$ is shorter than the diffusion timescale across that same spatial scale, the radiation acoustic mode can be supported by the medium. Thus, there is a critical diffusion rate defined by the rough inequality $\chi_{c, r}^{-1} \gtrsim \vartheta_{s}^{-1 / 2}$, for which the radiation acoustic mode exists. This criterion on the diffusion rate $\chi$ can be obtained from an approximate solution to equation (27) in the limit $\vartheta_{s} \gg 1$ and $\vartheta_{T} \rightarrow 0$, obtained by setting $\xi \approx 0$ for the radiation acoustic mode. I find that

$$
1 \approx \frac{\chi_{c, r}}{2 \vartheta_{s}^{1 / 2}} \approx \frac{2 \pi}{6}\left(\frac{c}{c_{s, r}}\right) \frac{1}{\tau_{k}}
$$

where $\tau_{k}=\kappa \rho\left(2 \pi k^{-1}\right)$ is the optical depth on a spatial scale $2 \pi k^{-1}$. Thus, if $\chi$ is small with respect to $\chi_{c, r}$, then the diffusion rate on a scale $k^{-1}$ is small compared to $c_{s, r} k$ and the radiation acoustic mode can be supported. Conversely, for $\chi \gtrsim \chi_{c, r}$, such a mode does not exist. Note that the critical value $\chi_{c, r}$ is decreased by nonzero $\vartheta_{T}$ (see eq. [31]) and modified if $\vartheta_{s}$ is larger than, but near, unity.

\subsection{Criterion Defining "Rapid" and "Slow" Diffusion}

Sections 2.2 and 2.3 distinguish between the limits of "rapid" and "slow" diffusion. The criterion that separates these two limits defines a critical diffusion rate $\chi_{c \text {,diff }}$ that can be estimated by setting the growth timescale for the unstable diffusion mode in equation (32) equal to unity, the inverse of the dynamical timescale. When $\vartheta_{s} \gg 1 \gg \vartheta_{T}$,

$$
\chi_{c, \text { diff }} \approx \vartheta_{s} .
$$

For $\chi>\chi_{c \text {,diff }}$ the medium is "rapidly" diffusing, and for $\chi<$

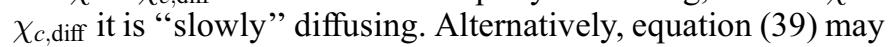
be written as (cf. eq. [37])

$$
1 \approx \frac{c k^{2}}{3 \kappa \rho} \frac{(4 \pi G \rho)^{1 / 2}}{c_{s, r}^{2} k^{2}} \Rightarrow t_{r} \approx\left(t_{\mathrm{diff}} t_{\mathrm{dyn}}\right)^{1 / 2}
$$

if the radiation pressure acoustic sound crossing timescale on a scale $k^{-1}$ is less than the geometric mean between the diffusion timescale on that same spatial scale and the dynamical timescale, then diffusion is "slow" and the stability properties of the medium are best described by $\S 2.3$. Conversely, if $t_{r}>$ $\left(t_{\text {diff }} t_{\mathrm{dyn}}\right)^{1 / 2}$, diffusion is "rapid" $(\S 2.2)$. This criterion is valid only at high- $k$ and in that regime is independent of spatial scale. Equations (38) and (39) imply that $\chi_{c \text {,diff }}$ can be greater than $\chi_{c, \mathrm{r}}$ and therefore that even though diffusion is "slow," the radiation acoustic mode is not supported.

\subsection{Solutions to the Dispersion Relation}

The limits of fast and slow diffusion, the criterion separating them, and the range of existence of the acoustic modes and their damping rates are illustrated in the solution to equation (27) presented in Figures 1 and 2, which show the modes $\xi$ obtained for a wide range of $\chi$, at fixed $\vartheta_{S}$ and $\vartheta_{T}$. Increasing $\chi$ while keeping $\vartheta_{s}$ and $\vartheta_{T}$ constant can be thought of as a continuous decrease in the opacity $\kappa$ at fixed $k^{-1}$. Open and filled circles show the real and imaginary part of $\xi$, respectively. Individual pieces of the various roots are labeled for comparison with equations (28)-(32).

The left panel of Figure 1 shows a case with $\vartheta_{s}>1$ and $\vartheta_{T}<1$. The unstable mode is the only positive imaginary root. For large $\chi$ it is the dynamical Jeans instability: $\xi \approx \pm i\left(1-\vartheta_{T}\right)^{1 / 2}$, whereas for small $\chi$ it is the diffusive mode of equation (32). The dotted line shows the approximation to $\chi_{c, r}$ (eq. [38]). Because for the parameters chosen, $\chi_{c, r} \approx 2 \vartheta_{s}^{1 / 2} \approx \vartheta_{s}$, the dotted line denoting $\chi_{c, r}$ also roughly corresponds to $\chi_{c \text {,diff }}$ (eq. [39]). For $\chi \lesssim \chi_{c, r}$, the radiation acoustic modes are evident and modestly damped. For any $\chi \neq 0$, the medium is unstable, because $\vartheta_{T}<1$. Note that the purely damped mode $\xi \approx-i \chi$ is off-scale for large $\chi$ (eq. [29]). Contrast the left panel of Figure 1 with the right panel, which shows the same calculation, but with $\vartheta_{T}=3 / 2>1$. Because $\vartheta_{T}>1$, the medium is stable for any $\chi$. For the parameters chosen, the adiabatic acoustic mode, which contains contributions from radiation and gas, joins smoothly into the isothermal gas acoustic mode at modest $\chi$.

Figure 2 presents a similar calculation, but more radiation pressure dominated, with $\vartheta_{s}=20$ and $\vartheta_{T}=1 / 10($ left $)$ and $\vartheta_{T}=$ $3 / 2(r i g h t)$. The left panel of Figure 1 is qualitatively identical to the left panel of Figure 2, but in the latter there is a clear separation between $\chi_{c, r}$ (dotted line) and $\chi_{c, \text { diff }}$ (dashed line). The right panel of Figure 2 again shows a case with $\vartheta_{T}>1$, so that the Jeans instability is stabilized for any $\chi$, but shows the separation in $\chi$ between the adiabatic acoustic mode, which is here highly radiation pressure dominated $\left(\chi \lesssim \chi_{c, r}\right)$, and the 

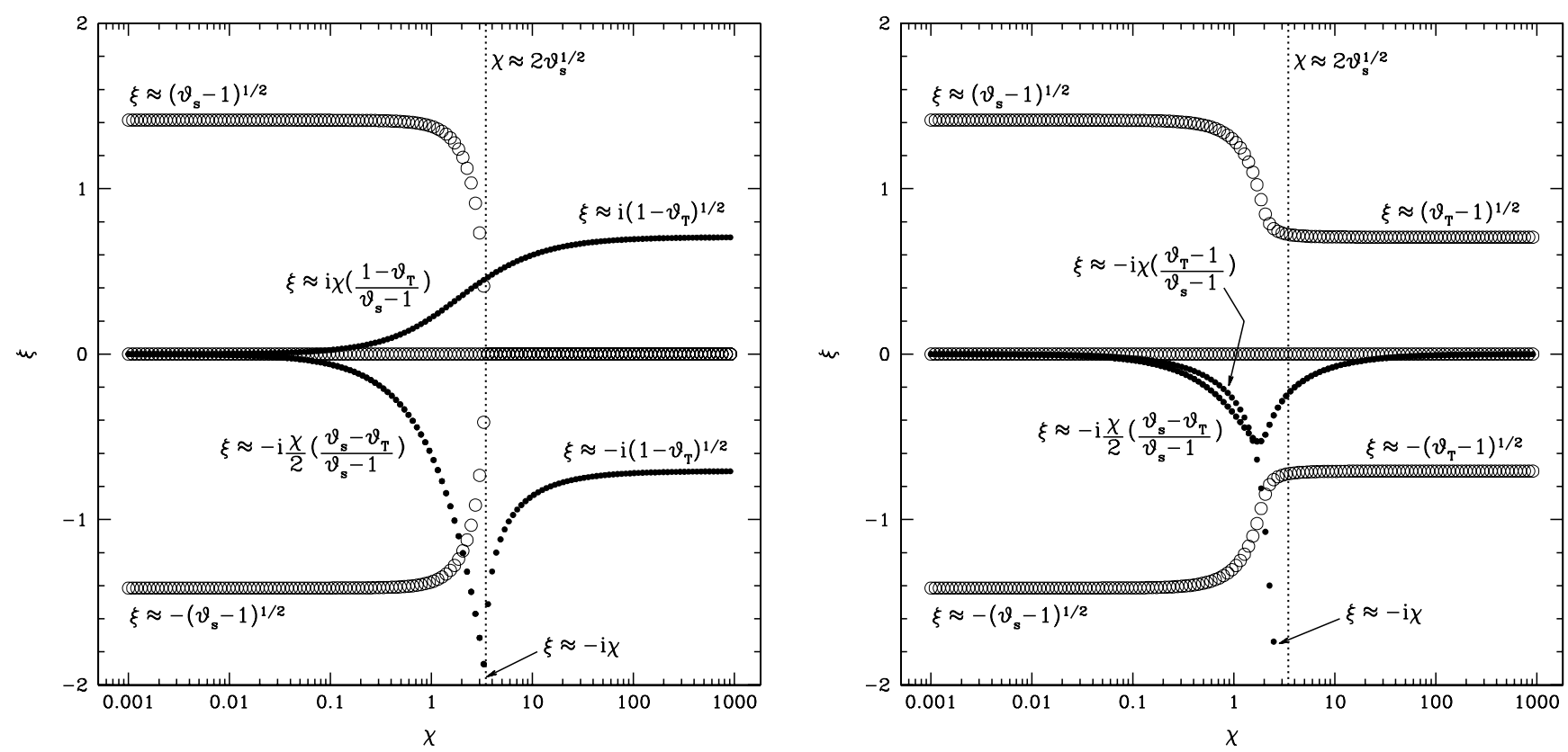

FIG. 1. - Left: Solution to eq. (27) for $\vartheta_{s}=3$ and $\vartheta_{T}=1 / 2$, for $10^{-3} \leq \chi \leq 10^{3}$ at fixed $k^{-1}$. Open and filled circles show the real and imaginary parts of the three roots $\xi$, respectively. Positive complex roots indicate instability. Because $\vartheta_{T}<1$ on the scale chosen, the medium is unstable any $\chi \neq 0$. For large $\chi$ the instability is dynamical, whereas for small $\chi$ the medium is unstable to the diffusive instability given by eq. (32). The dotted line shows the approximate solution for $\chi_{c, r}$ (eq. [38]),

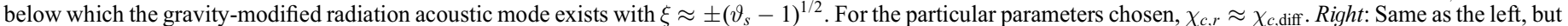
for $\vartheta_{T}=3 / 2$. Because $\vartheta_{T}$ is larger than unity, the gravitational instability is stabilized for all $\chi$. Note the transition from adiabatic (radiation plus gas) to isothermal (gas only) gravity-modified acoustic waves.

damped gravity-modified isothermal gas acoustic mode that exists for $\chi \gtrsim\left(\vartheta_{s}-\vartheta_{T}\right) / 2\left(\vartheta_{T}-1\right)^{1 / 2}$ (dashed line $)$.

Although I do not plot it here, equations (28)-(32) and the general solution to equation (27), show that in the limit $\vartheta_{T} \rightarrow 0$ and $\vartheta_{s} \rightarrow 0$ the medium is dynamically unstable for any $\chi$.

\subsection{The Connection to Treatments of Silk Damping}

The calculation of the dispersion relation and the slow diffusion limit of $\S 2.3$ is most closely related to the nonrelativistic calculation of Silk (1967) in the context of the early universe. His expression for the damping rate of adiabatic radiation-dominated acoustic modes is essentially equivalent to the damping term in equation (31), without the " 1 " in the denominator and with $\vartheta_{T} \rightarrow 0$. The correct relativistic expression for the damping rate was subsequently obtained by Weinberg (1971). The qualitative difference here with respect to Silk (1967) and other versions of the derivation of the damping of acoustic modes in the early universe by radiative diffusion (see, e.g., Hu \& Sugiyama 1996) is equation (32), which shows that the medium is unstable to a slow diffusive mode precisely in the regime $\left(\vartheta_{s}>1\right)$ where the medium is stable to the adiabatic Jeans criterion - that is, on scales below the adiabatic Jeans length (eq. [25]). A calculation of the dispersion relation in the cosmological context (in an expanding background) and an evaluation of the importance of this mode is in preparation ( T. A. Thompson 2008, in preparation). If it grows, it should do so very slowly on a timescale many times the dynamical timescale, and it should be purely nonadiabatic. Although I have not done a relativistic calculation here, taking $c_{s, r}^{2}=c^{2} / 3$ in equation (33), I find that $\omega \approx i 4 \pi G /(\kappa c)$. This is the inverse of the Kelvin-Helmholtz timescale for a relativistic radiation pressure supported self-gravitating object. ${ }^{3}$ The characteristic timescale for growth is of order $t_{\mathrm{KH}} \sim 10^{16}\left(\kappa / 0.4 \mathrm{~cm}^{2} \mathrm{~g}^{-1}\right) \mathrm{s}$, which is of order thousands of times longer than the age of the universe at

\footnotetext{
${ }^{3}$ Note the correspondence with the Salpeter timescale for black hole growth.
}

decoupling. Because the total matter density (which sets the gravitational driving term, the numerator of eq. [33]) is roughly 10 times the baryon density (which sets the scattering timescale, the denominator of eq. [33]), one expects a more careful derivation to yield a timescale shorter by this ratio.

\subsection{Extensions}

Motivated by Chandrasekhar (1961), Appendix A contains an analysis analogous to $\S 2$, but including uniform rotation in the background medium. As in the case with only gas pressure, modes propagating at right angles to the angular momentum vector are stabilized by rotation if the angular velocity $(\Omega)$ is large enough that $\Omega^{2}>\pi G \rho$ (see also Goldreich \& Lynden-Bell 1965).

Appendix B accounts for the time dependence of the radiation field and the possibility of imperfect energetic coupling between the radiation field and the gas. For the parameters appropriate to the astrophysical applications discussed in $\S 3$ these factors are largely unimportant for the qualitative stability properties of the medium. This follows from the fact that the characteristic frequency for energetic coupling between the radiation and the gas, $\omega_{\text {th }} \approx \kappa \rho c\left(u_{r} / u_{g}\right)$ (see eq. [B8] and surrounding discussion, as well as Bogdan et al. 1996; Blaes \& Socrates 2003), is likely to be large in the contexts considered. As in the work of, e.g., Dzhalilov et al. (1992), Zhugzhda et al. (1993), and Bogdan et al. (1996), yet more precise descriptions of the transport should be explored, as should the dependence of the stability properties on the temperature and density dependence of the opacity (e.g., BisnovatyiKogan \& Blinnikov 1979; Zhugzhda et al. 1993; Blaes \& Socrates 2003) and the explicit dependence on the scattering albedo (e.g., Kaneko \& Morita 2006).

Gradients in the background state - and, particularly, a background flux - have been neglected in the analysis of $\S 2$. Ledoux (1951) considered a consistent background state without invoking the Jeans swindle in calculating the Jeans instability and found only a small quantitative change to the stability properties of the 

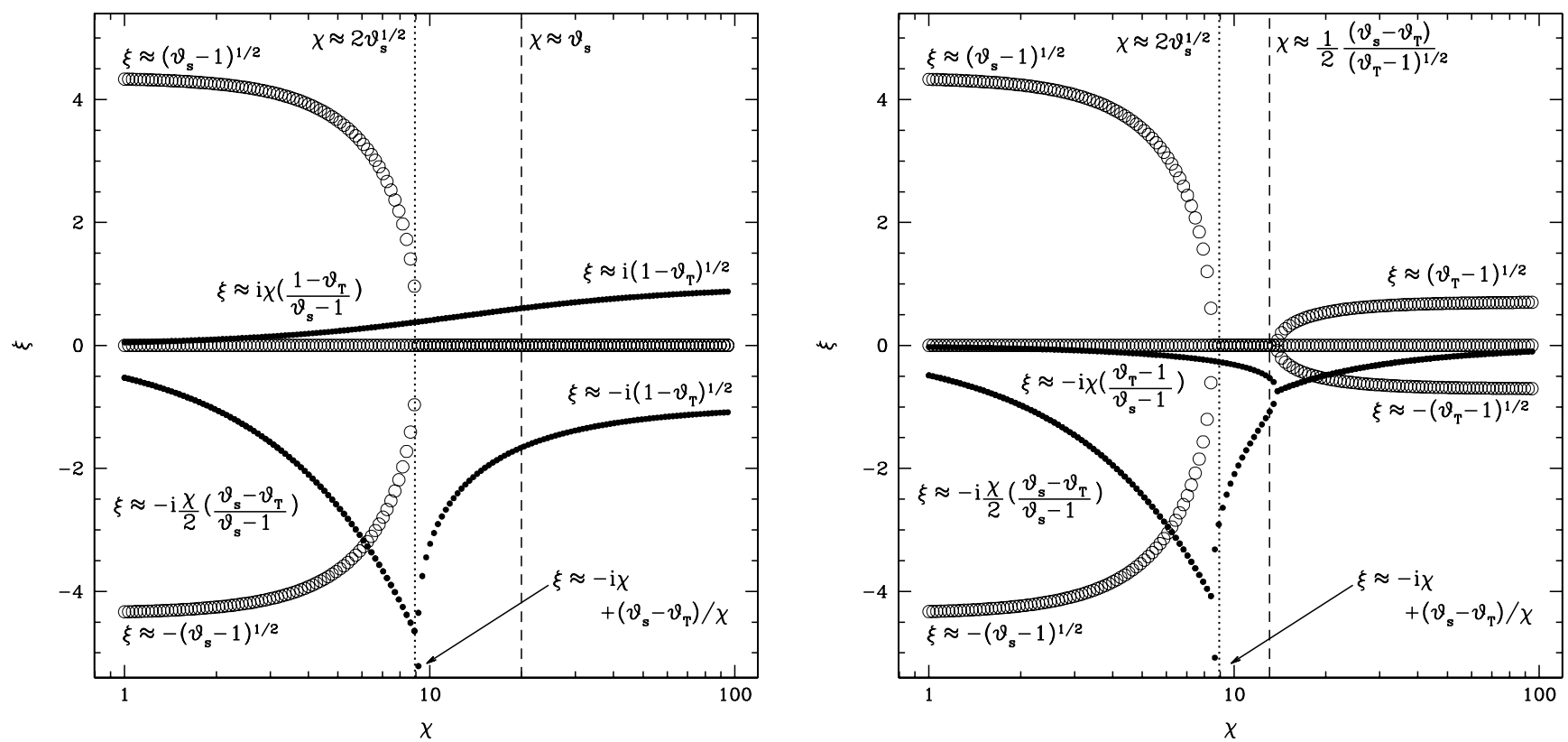

FIg. 2.-Left: Same as Fig. 1, but for $\vartheta_{s}=20$ and $\vartheta_{T}=1 / 10$, for $1 \leq \chi \leq 10^{2}$ at fixed $k^{-1}$. Again, because $\vartheta_{T}<1$ on the scale chosen, the medium is unstable for

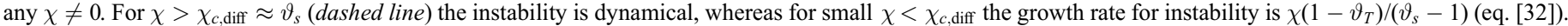
The dotted line denotes $\chi_{c, r}$ (eq. [38]). Right: Same as the left, but $\vartheta_{T}=3 / 2$. Because $\vartheta_{T}$ is larger than unity, the medium is stable for all $\chi$. The dashed line denotes the critical $\chi$ above which the isothermal gas acoustic wave exists with $\xi \approx \pm\left(\vartheta_{T}-1\right)^{1 / 2}$.

medium: the Jeans length was increased by a factor of $\sqrt{2}$. A detailed assessment of such gradients in the context of radiation pressure-dominated self-gravitating media, as well as an exploration of magnetic fields and the physics of the photon bubble instability (Arons 1992; Gammie 1998; Blaes \& Socrates 2001, 2003) are saved for a future effort.

\section{DISCUSSION}

The analysis of $\S 2$ indicates that under the assumption of optically thick equilibrium radiative diffusion, an isotropic selfgravitating medium is unstable if the classical gas-only isothermal Jeans criterion is not satisfied. If diffusion is rapid, the instability is dynamical - the classical Jeans instability. If diffusion is slow, then on scales larger than the adiabatic Jeans length the medium is dynamically unstable - again, the Jeans instability. However, on scales smaller than the adiabatic Jeans length the medium is unstable to a diffusive mode that acts on a timescale of order the Kelvin-Helmholz timescale (eq. [33]), longer than the dynamical timescale of the medium.

Depending on whether or not the medium is rapidly or slowly diffusing (cf. eqs. [39] and [40]), the growth time for gravitational instability may be significantly decreased with respect to the dynamical timescale corresponding to the average density of the medium. Here I discuss several astrophysical environments where this analysis is applicable and where it provides some insight into the stability properties of the medium.

\subsection{Radiation in Starbursts, ULIRGs, Parsec-Scale AGN Disks, and Extreme Massive Star-forming Regions}

Starburst galaxies are marked by high radiation energy density, high gas density, and optical depths to their own dust-reprocessed infrared radiation that exceed unity. Scoville et al. (2001), Scoville (2003), and TQM05 have argued that radiation pressure may dominate the dynamics in these systems. Typical temperatures are in the range $T \sim 50-200 \mathrm{~K}$ and densities range from $n \sim$ $500-10^{3} \mathrm{~cm}^{-3}$ (e.g., the central regions of M82 and NGC 253) to $n \gtrsim 2 \times 10^{4} \mathrm{~cm}^{-3}$ (e.g., the nuclei of the ULIRG Arp 220;
Downes \& Solomon 1998) on $\sim 100$ pc scales. In extreme massive star-forming regions temperatures are similar to those in starburst galaxies and ULIRGs, but the typical density of clumps and cores responsible for star formation can be higher. An example is the core of NGC 5253 with $n \approx 10^{7} \mathrm{~cm}^{-3}$ and a physical scale of order 1 pc (e.g., Turner et al. 2000).

Yet more extreme physical conditions are expected to obtain in the self-gravitating parsec-scale disks or obscuring "tori" thought to attend the process of fueling active galactic nuclei (e.g., Pier \& Krolik 1994; Goodman 2003; Sirko \& Goodman 2003; TQM05). There, one expects central disk temperatures approaching the sublimation temperature of dust grains, $T_{\text {sub }} \sim 10^{3} \mathrm{~K}$. Although the gas density is uncertain in these environments, we can make an estimate by assuming that the disk is marginally Toomre-stable such that $n \approx \Omega^{2} /\left(2 \pi G m_{p}\right) \approx 6 \times 10^{8} M_{8} R_{1 \mathrm{pc}}^{-3} \mathrm{~cm}^{-3}$, where $M_{8}=M / 10^{8} M_{\odot}$ and $R_{1 \mathrm{pc}}=R / 1 \mathrm{pc}$, within the sphere of influence of the central supermassive black hole. ${ }^{4}$

Simple estimates indicate that the dust and gas in these systems are collisionally and energetically coupled and that the IR optical depth is larger than unity. Ignoring the enhancement of dust-gas coupling due to magnetic fields and grain charging, the mean free path for momentum coupling is

$$
\lambda_{d g} \approx 10^{-3} n_{4}^{-1} \mathrm{pc} \approx 10^{-7} n_{8}^{-1} \mathrm{pc},
$$

where $n_{x}=n / 10^{x} \mathrm{~cm}^{-3} .{ }^{5}$ The medium is optically thick to the dust-reprocessed IR radiation field on scales larger than

$$
\begin{aligned}
\lambda_{\tau=1} & =(\kappa \rho)^{-1} \approx 8 \kappa_{2.5}^{-1} n_{4}^{-1} \mathrm{pc} \\
& \approx 8 \times 10^{-4} \kappa_{2.5}^{-1} n_{8}^{-1} \mathrm{pc},
\end{aligned}
$$

\footnotetext{
${ }^{4}$ The sublimation radius for dust is $R_{\text {sub }} \approx 1 L_{46}^{1 / 2} T_{3}^{-2} \mathrm{pc}$, where $T_{3}=T_{\text {sub }} / 10^{3} \mathrm{~K}$ and $L_{46}=L_{\mathrm{BH}} / 10^{46} \mathrm{ergs} \mathrm{s}^{-1}$ is the Eddington luminosity for a $10^{8} M_{\odot}$ black hole.

${ }^{5}$ Here, I have assumed a dust grain density of $3 \mathrm{~g} \mathrm{~cm}^{-3}$ and an average dust grain radius of $a_{d} \approx 0.1 \mu \mathrm{m}$. Because grain charging and magnetic fields are likely to be important the dust and gas may be regarded as a single, coupled fluid on scales larger than $\lambda_{d g}$.
} 
where $\kappa_{2.5}=\kappa / 2.5 \mathrm{~cm}^{2} \mathrm{~g}^{-1}$ is a representative Rosseland-mean dust opacity for $T \approx 100 \mathrm{~K}$, assuming solar metallicity and a Galactic dust-to-gas ratio (e.g., Fig. 1 from Semenov et al. 2003). For temperatures near the dust sublimation temperature, $\kappa_{2.5} \approx 1$ is also a fair order-of-magnitude approximation (but see Chang et al. 2007). Because $\lambda_{\tau=1} / \lambda_{d g} \approx 8 \times 10^{3} / \kappa_{2.5} \gg 1$, the dust and gas are always highly collisionally coupled if the average medium is optically thick. A rough estimate of the optical depth in the nuclei of the ULIRG Arp 220, where the scale of the system is $R \sim 100 \mathrm{pc}$, is $\tau_{\mathrm{IR}} \approx 10 \kappa_{2.5} n_{4} R_{100 \mathrm{pc}}$. In systems like M82 and NGC 253, $n$ is lower and the medium is only marginally optically thick on $100 \mathrm{pc}$ scales. Although observations indicate that the obscuring material surrounding AGN may occupy a large fraction of $4 \pi$, theoretical arguments suggest that most of the gas may be confined to a thin disk with vertical scale height $h \ll R$ (e.g., Thompson et al. 2005; Chang et al. 2007; Krolik 2007). The vertical optical depth in such a disk is then $\tau_{\mathrm{IR}} \approx 10^{2} \kappa_{2.5} n_{8}(h / 0.1 \mathrm{pc})$.

Although the dust-gas fluid is highly collisionally coupled on the scales of interest, the energetic coupling may not be perfect. However, in regions for which the cooling line radiation is optically thick, we do not expect large temperature differences between the gas and dust, similar to the case in dense molecular clouds where the gas temperature is maintained by a combination of heating by dust-gas collisions and cosmic rays, and cooling in molecular lines (see, e.g., Gorti \& Hollenbach 2004). At high density, inelastic dust-gas collisions likely dominate gas heating. Assuming order-unity differences between the gas and dust temperatures, the gas heating timescale is roughly

$$
t_{\text {heat }} / t_{\text {dyn }} \approx 0.03 n_{4}^{-1 / 2} T_{2}^{-1 / 2} \approx 10^{-4} n_{8}^{-1 / 2} T_{3}^{-1 / 2},
$$

where here $t_{\mathrm{dyn}}=(G \rho)^{-1 / 2}$. Assuming tight dust-gas coupling and $\tau_{\mathrm{IR}} \gtrsim 1$, the medium is highly radiation pressure dominated:

$$
p_{r} / p_{g} \approx 10^{3} T_{2}^{3} n_{4}^{-1} \approx 10^{2} T_{3}^{3} n_{8}^{-1} .
$$

Although these estimates imply that starbursts and AGN disks are optically thick and potentially modestly to very strongly radiation pressure dominated, the ratio ${ }^{6}$

$$
\begin{aligned}
\frac{\chi}{\vartheta_{s}} & \approx \frac{(4 \pi G \rho)^{1 / 2}}{\left(4 p_{r} \kappa / c\right)} \approx 1400 \kappa_{2.5}^{-1} n_{4}^{1 / 2} T_{2}^{-4} \\
& \approx 14 \kappa_{2.5}^{-1} n_{8}^{1 / 2} T_{3}^{-4}
\end{aligned}
$$

shows explicitly that diffusion of radiation in starbursts is very "rapid." The stability properties of these media on the scale of the system are thus best represented by the far right-hand portion of the left panels of Figures 1 and 2: gas pressure is negligible and radiation pressure is important as measured by the adiabatic Jeans number $\vartheta_{s}$, but $\chi \gg \vartheta_{s}$ and the medium is dynamically (Jeans) unstable.

For fiducial parameters, the scaling for AGN disks also indicates that they are rapidly diffusing. However, the disk parameters in this regime are quite uncertain. For example, Chang et al. (2007) advocate $\kappa_{2.5} \approx 20$ or larger for a gas density $n \sim 10^{8} \mathrm{~cm}^{-3}$ and solar metallicity, implying $\chi / \vartheta_{s} \sim 0.7 T_{3}^{-4}$. The linear dependence of $\kappa$ on metallicity and the strong temperature dependence of $\chi / \vartheta_{s}$ implies that if grains persist for $T \gtrsim 10^{3} \mathrm{~K}$ and/or the composition of the disk is supersolar, the medium may transition to slowly diffusing, and the timescale for gravitational instability will be increased to $t_{\mathrm{KH}}$ (eqs. [33] and [37]). Also, an estimate of

\footnotetext{
${ }^{6}$ Here, $\left(A / C_{V}\right)$ in $\chi$ is taken as $\approx 1$ (see eqs. [20] and [26]).
}

$\vartheta_{T}$ shows that gas pressure becomes important on small scales in AGN disks and may stabilize the medium in the linear regime (TQM05).

\subsubsection{The Nonlinear Outcome}

Consider an initial hypothetical equilibrium configuration for a self-gravitating disk with starburst/ULIRG-like characteristics such that $\vartheta_{T} \ll 1, \vartheta_{s} \approx 1$ on $\sim 100$ pc scales and with $c_{s}^{2} \approx$ $c_{s, r}^{2} \approx p_{r} / \rho \approx(h \Omega)^{2}$, where $h$ is the disk scale height (as in the models of TQM05). Because diffusion is "rapid," the configuration is dynamically unstable on all scales larger than the classical gas Jeans length $(\ll h)$ and vertical hydrostatic equilibrium cannot be maintained in the linear regime. ${ }^{7}$

The nonlinear outcome of the Jeans instability in such a system is highly uncertain in part because it is tied to star formation, which, in turn, determines the character of the radiation field. The question of whether or not hydrostatic equilibrium can be maintained depends crucially on the nonlinear coupling of the radiation and the gas. One possibility is that the large-scale radiation field produced by star formation is coupled to the generation of turbulence, which regulates the structure of the galaxy and its stability properties. Supersonic turbulence on large scales has been shown to inhibit the Jeans instability and gravitational collapse (e.g., Klessen et al. 2000; Mac Low \& Klessen 2004). It has also recently been invoked as a basis for understanding the origin of the Schmidt/Kennicutt laws (Kennicutt 1998; Krumholz \& McKee 2005). Indeed, the turbulent velocities inferred in local starbursts and ULIRGs are large enough that a "turbulent Jeans number," $\vartheta_{\text {turb }} \approx \delta v^{2} k^{2} /(4 \pi G \rho)$, analogous to $\vartheta_{s}$ and $\vartheta_{T}$, may indicate marginal stability: $\vartheta_{\text {turb }} \approx 1$. Thus, if radiation pressure forces can generate turbulence, perhaps a statistical hydrostatic equilibrium can be maintained.

There are at least two reasons why - in the absence of energetic input to the ISM from stars (e.g., supernovae) - star formation may be coupled to the generation of turbulence. First, because in the initial fictitious equilibrium state envisioned radiation pressure is large enough that $p_{r} / \rho \approx(h \Omega)^{2}$, the radiation field is capable of driving mass motions with velocities of order $\delta v \approx h \Omega$ if order-unity spatial variations in the radiation field are present. Second, although I have not shown it in this paper, one expects the astrophysical environments described here to be subject to the self-gravitating analog of the photon bubble instability, which in its nonlinear state will drive turbulence (Turner et al. 2005). The latter is particularly interesting because it motivates a dynamical coupling between the turbulent energy density $\left(u_{\text {turb }}\right)$, the photon energy density $\left(u_{\mathrm{ph}}\right)$, and the magnetic energy density $\left(u_{B}\right)$.

The second of these connections, between $u_{\mathrm{ph}}$ and $u_{B}$ in galaxies, can be motivated phenomenologically. Recently Thompson et al. (2006) have shown that the magnetic field strengths in starbursts significantly exceed estimates derived from the "minimum energy argument." In addition, they show that $u_{B}$ must be a constant, order unity, multiple of $u_{\mathrm{ph}}$ in these systems (see Condon et al. 1991). This conclusion follows from the linearity of the FIR-radio correlation, the radio spectral indices of star-forming galaxies at $\mathrm{GHz}$ frequencies, and the fact that the ratio $u_{B} / u_{\mathrm{ph}}$ measures the importance of synchrotron versus inverse Compton cooling of the cosmic-ray electrons and positrons (e.g., Condon 1992). The fact the galaxies that comprise the FIR-radio correlation have $u_{\mathrm{ph}}$-values that span five to six dex, and that in the Galaxy $u_{\mathrm{ph}} \approx u_{B}$, implies that $u_{B}$ must increase with $u_{\mathrm{ph}}$, from normal Milky Way-like galaxies to ULIRGs. The necessity of

\footnotetext{
7 The equilibrium imagined is likely also unstable to convective, magnetorotational, and photon-bubble instabilities (see Blaes \& Socrates 2001, 2003).
} 
this lock-step increase in both $u_{\mathrm{ph}}$ and $u_{B}$ may signal a dynamical coupling between the radiation field and the magnetic field in galaxies. Thus, the fact that $u_{\mathrm{ph}}, u_{B}$, and $u_{\text {turb }}$ are of the same order of magnitude may not be a coincidence, but instead a necessary consequence of the dynamical coupling between the radiation field and the self-gravitating magnetized ISM.

\subsection{Cosmic Rays in Normal Star-forming Galaxies and Clusters}

Although no attempt is made here to model the diffusion of cosmic rays, it is instructive to consider the various parameters governing gravitational stability in the case of cosmic rays vis à vis radiation.

The total pressure in cosmic rays in the Galaxy is $p_{\text {cr. } \mathrm{Mw}} \approx$ $10^{-12} \mathrm{ergs} \mathrm{cm}^{-3}$ (e.g., Boulares \& Cox 1990), comparable to the energy density in starlight, magnetic fields, and turbulence. The cosmic-ray lifetime is inferred to be $t_{\mathrm{cr}} \approx(2-3) \times 10^{7} \mathrm{yr}$ (GarciaMunoz et al. 1977; Connell 1998). Interpreted as a diffusion timescale on kpc scales, one infers a cosmic-ray scattering mean free path of order $l_{\mathrm{mfp}} \approx 0.1-1 \mathrm{pc}$. In addition, from the observed grammage traversed by cosmic rays in the Galaxy, one infers an average gas density encountered by the cosmic rays of $n \approx 0.2 \mathrm{~cm}^{-3}$ (Engelmann et al. 1990). Writing $l_{\mathrm{mfp}}=(\kappa \rho)^{-1}$ (cf. eq. [45]; see also Kuwabara \& Ko 2004), $\vartheta_{s} \approx 50 p_{12} n_{0.2}^{-2} \lambda_{\mathrm{kpc}}^{-2}$ and $^{8}$

$$
\frac{\chi}{\vartheta_{s}} \approx \frac{c \rho l_{\mathrm{mfp}}}{4 p_{\mathrm{cr}}}(4 \pi G \rho)^{1 / 2} \approx 0.4 l_{0.1 \mathrm{pc}} p_{\mathrm{cr}, \mathrm{MW}}^{-1} n_{0.2}^{3 / 2} .
$$

Although the parameters are uncertain, equation (46) indicates that cosmic rays are marginally slowly diffusing in normal starforming galaxies. Thus, as for photons in the dense parsec-scale AGN disks discussed in $\S 3.1$, perhaps on kpc scales $\chi / \vartheta_{s}$ may be somewhat less than unity so that the growth rate for the gravitational instability is (cf. eq. [37])

$$
t_{\mathrm{KH}} / t_{\mathrm{dyn}} \sim 3 p_{\mathrm{cr}, \mathrm{MW}} l_{0.1 \mathrm{pc}}^{-1} n_{0.2}^{-1 / 2}
$$

Depending on the phase of the ISM considered, the isothermal Jeans number $\vartheta_{T}$ may be very close to unity so that the critical $\chi$ below which the Jeans instability is suppressed can be made significantly larger (eq. [32]). Indeed, cosmic rays have recently been proposed as an important large-scale feedback mechanism in star-forming galaxies (Socrates et al. 2008).

Similar estimates may be written down for the central regions of galaxy clusters, but it is unclear if these regions may plausibly be cosmic-ray pressure dominated. If they are at least modestly so, scaling from equation (46) for higher pressures and lower densities, we see then that for $0.1 \lesssim l_{\mathrm{mfp}} \lesssim 100 \mathrm{pc}$ they are plausibly in the "slow" diffusion limit $\left(\chi / \vartheta_{s}<1\right)$; again, the dynamical Jeans instability is quelled by the nonthermal pressure support. In this limit, the diffusive instability identified in equation (32) still acts on $t_{\mathrm{KH}}$, but this timescale is likely many times the age of the universe: for $c_{s, r} \approx 1000 \mathrm{~km} \mathrm{~s}^{-1}$ and $n \approx 10^{-2}, t_{\mathrm{KH}} \approx 10^{12}\left(l_{\mathrm{mfp}} / \mathrm{pc}\right) \mathrm{yr}$.

\subsection{Individual Massive Stars}

Individual massive stars are radiation pressure dominated and slowly diffusing and may in principle also be subject to the

\footnotetext{
${ }^{8}$ For simplicity, here I take $A / C_{V}=1$ in $\chi$. This is a rough approximation in the context of the Galaxy because, depending on which gas phase of the ISM is being considered, $\vartheta_{T}$ may be the same order of magnitude as $\vartheta_{s}$.
}

diffusive mode identified in $\S 2$. This is simply a secular instability of the kind discussed in, e.g., Hansen (1978, and references therein). If present, the growth timescale is the KelvinHelmholz time, of order $t_{\mathrm{KH}} \sim 3 \kappa c_{s, r}^{2} /(4 \pi G c) \sim 10^{3}$ yr for typical parameters, where $\kappa$ is the Thomson opacity and $c_{s, r}$ is the adiabatic radiation pressure-dominated sound speed of the fluid $\sim(G M / R)^{1 / 2}$. Although massive stars are known to be globally secularly unstable on the Kelvin-Helmholtz timescale, it is possible that otherwise stably stratified (radiative) regions of their interiors may be locally unstable to a variant of the diffusive instability in equation (33).

\section{SUMMARY AND CONCLUSION}

I consider the physics of gravitational instabilities in the presence of dynamically important radiation pressure and radiative diffusion. I find that the medium is always stable on scales smaller than the gas-only isothermal Jeans length, $\lambda_{\mathrm{J}, T}$ (eq. [23]). For scales larger than $\lambda_{\mathrm{J}, T}$ there are two possibilities, depending on whether the medium is "slowly" or "rapidly" diffusing, as defined in $\S 2.5$. When diffusion is rapid, radiation leaks out of a perturbation without providing a sufficient restoring pressure force and the medium is dynamically unstable on all scales larger than $\lambda_{\mathrm{J}, T}$, regardless of the dominance of radiation pressure.

The limit of slow diffusion is more interesting. Here, the medium is unstable to a diffusive mode at an intermediate range of scales between the gas-only isothermal Jeans length $\lambda_{\mathrm{J}, T}$ and the larger (gas + radiation) adiabatic Jeans length $\lambda_{\mathrm{J}, s}$ (eq. [25]). The characteristic growth timescale is longer than the dynamical timescale. Neglecting gas pressure, it is given approximately by equation (33) (see also eqs. [32] and [37]), which is simply the Kelvin-Helmholz timescale for a radiation pressure-supported self-gravitating fluid parcel to radiate its total thermal energy at the Eddington limit. Note that on small spatial scales, the characteristic timescale is independent of scale. For $\lambda>\lambda_{\mathrm{J}, s}$ the medium is dynamically "Jeans" unstable, as expected. Thus, even when radiation pressure is dynamically dominant, on precisely the scales where the medium is dynamically stable by the usual Jeans criterion $\left(\lambda<\lambda_{\mathrm{J}, s}\right)$, it is unstable to a diffusive instability that operates on the Kelvin-Helmholtz time. I conclude that radiation cannot formally stabilize a self-gravitating medium on scales larger than the gas-only isothermal Jeans length. See also the discussion of Kaneko \& Morita (2006).

In $\S 3.1$, I consider the importance of the results derived in $\S 2$ for extreme sites of massive star formation including starburst galaxies and parsec-scale AGN disks. I argue that the average medium in these systems is likely to be radiation pressure dominated and optically thick. Importantly, for fiducial parameters the photons in these systems are in the rapidly diffusing limit $\left(\chi / \vartheta_{s}>\right.$ 1 ; eq. [45]). For fairly extreme choices for the uncertain physical parameters in parsec-scale AGN disks (e.g., the opacity $\kappa$ ) this environment is marginally slowly diffusing and thus the stability properties of the medium might be qualitatively different from rapidly diffusing starbursts.

TQM05 developed a theory of marginally Toomre-stable starburst and AGN disks supported by feedback from radiation pressure. Because the IR photons produced by dust-reprocessed starlight in these systems diffuse rapidly and because their characteristic sizes are much larger than the classical gas-only Jeans length, the analysis presented here dictates that they cannot be supported in the linear regime by radiation pressure alone. One may wonder, then, why the entire mass of gas in starbursts does not fragment into stars on a single dynamical time, in apparent contradiction with observations (e.g., Kennicutt 1998). If radiation pressure is to be the dominant feedback mechanism, then the 
answer must be that these forces are coupled to the generation of supersonic turbulence, which may mitigate against complete collapse and fragmentation on scales larger than the gas-only Jeans length. In $\S 3.1 .1 \mathrm{I}$ argue that the generation of turbulence likely proceeds from the nonlinear coupling of the Jeans instability with the radiation field through star formation, and may be driven by the self-gravitating analog of the photon bubble instability. This may help explain the apparent order-of-magnitude equivalence between the radiation, magnetic, and turbulent energy densities in starburst systems. Thus, it is important to emphasize that the conclusion that radiation pressure alone cannot stave off gravitational instability in the rapidly diffusing limit does not necessarily imply that the disk cannot be maintained in global hydrostatic equilibrium in an average sense by radiation pressure in the nonlinear, turbulent regime.

In $\S 3.2$, I consider the case of cosmic rays diffusing in the Galaxy and the cosmic-ray halo, and in galaxy clusters. Although no attempt is made here to calculate the physics of cosmic-ray diffusion and their thermal coupling to the gas, they provide a useful point of contrast with radiation because of their very high scattering optical depths. Even so, this constituent of the ISM of the Galaxy is at the border of "slow" and "rapid" diffusion $\left(\chi / \vartheta_{s} \approx 1\right)$ outlined in $\S 2$ (eq. [46]) for fiducial parameters. In the cluster context diffusion is likely more fully in the "slow" limit, but it is unclear if cosmic rays dominate the total pressure budget in the central regions (e.g., Guo \& Oh 2008). Finally, I also briefly mention the possibility that individual massive stars may be locally unstable to this diffusive mode on the local KelvinHelmholz timescale in otherwise stably stratified radiative regions of their interiors.

This paper was motivated in part by stimulating conversations with Aristotle Socrates. I also thank Yoram Lithwick, Andrew Youdin, Charles Gammie, Kristen Menou, and Jeremy Goodman for several useful conversations and Eliot Quataert, Norm Murray, Bruce Draine, and Julian Krolik for encouragement. Finally, I am grateful to the Department of Astrophysical Sciences at Princeton University, where much of this work was completed. This paper is dedicated to Garnett A. B. Thompson.

\section{APPENDIX A}

\section{UNIFORM ROTATION}

Chandrasekhar $(1954,1961)$ explored the effect of uniform rotation on the Jeans instability and found that the dispersion relation is modified by the Coriolis force in the rotating frame. In particular, he showed that for the special case of waves propagating at right angles to the direction $\hat{\Omega}\left(\cos ^{2} \theta=0\right)$ that equation (1) becomes $\omega^{2}=4 \Omega^{2}+c_{g}^{2} k^{2}-4 \pi G \rho-$ that is, if $\Omega^{2}>\pi G \rho$, then the Jeans instability is stabilized for any gas sound speed $c_{g}\left(\delta p_{g}=c_{g}^{2} \delta \rho\right.$ assumed). For the general case $\cos ^{2} \theta \neq 0$-for waves whose wavevectors have arbitrary angles with respect to the spin axis - if the classical gas Jeans criterion (eq. [2]) indicates instability $\left(\vartheta_{T}<1\right)$, then the medium is unstable for any $|\Omega|$. In order to gain some intuition and to make contact with the work of Chandrasekhar (1954) it is useful to consider the Jeans instability including uniform rotation, radiation pressure, and radiative diffusion. The equation expressing conservation of momentum in the rotating frame is

$$
\frac{\partial \boldsymbol{v}}{\partial t}+\boldsymbol{v} \cdot \nabla \boldsymbol{v}=-\frac{1}{\rho} \nabla P-\nabla \Phi+2(\boldsymbol{v} \times \Omega) .
$$

All other equations in the original analysis of $\S 2$ are unchanged. I take $\boldsymbol{k}=\left(0,0, k_{z}\right)$ and $\Omega=\left(0, \Omega_{y}, \Omega_{z}\right)$. The perturbation equations in component form are

$$
\begin{gathered}
-i \omega \delta \rho+i \rho k_{z} \delta v_{z}=0, \\
-i \omega \delta v_{x}-2 \delta v_{y} \Omega_{z}+2 \delta v_{z} \Omega_{y}=0, \\
-i \omega \delta v_{y}+2 \delta v_{x} \Omega_{z}=0 \\
-i \omega \delta v_{z}+i k_{z} \delta P / \rho+i k_{z} \delta \Phi-2 \delta v_{x} \Omega_{y}=0, \\
-i \omega \delta U+(U+P) i k_{z} \delta v_{z}+i k_{z} \delta F_{z}=0, \\
-k^{2} \delta \Phi-4 \pi G \delta \rho=0, \\
i k_{z} \delta u_{r}+3 \kappa \rho \delta F_{z} / c=0 .
\end{gathered}
$$

The resulting dispersion relation is (cf. eq. [19])

$$
\begin{aligned}
\omega^{5}+i \tilde{\omega} \omega^{4}-\omega^{3}\left(4 \Omega^{2}+c_{s}^{2} k^{2}-4 \pi G \rho\right)-i \tilde{\omega} \omega^{2}\left(4 \Omega^{2}+\right. & \left.c_{T}^{2} k^{2}-4 \pi G \rho\right) \\
& +\omega\left(4 \Omega^{2} \cos ^{2} \theta\right)\left(c_{s}^{2} k^{2}-4 \pi G \rho\right)+i \tilde{\omega}\left(4 \Omega^{2} \cos ^{2} \theta\right)\left(c_{T}^{2} k^{2}-4 \pi G \rho\right)=0,
\end{aligned}
$$

where $\cos \theta=\Omega_{z} /|\Omega|$ and $\tilde{\omega}$ is the radiation diffusion rate given in equation (17). Defining

$$
Q=\Omega^{2} /(\pi G \rho)
$$

and using the definitions for $\vartheta_{T}, \vartheta_{s}, \chi$, and $\xi$, in equations (22)-(26), equation (A2) can be rewritten as

$$
\xi^{5}+i \chi \xi^{4}-\xi^{3}\left(Q+\vartheta_{s}-1\right)-i \chi \xi^{2}\left(Q+\vartheta_{T}-1\right)+\xi Q \cos ^{2} \theta\left(\vartheta_{s}-1\right)+i \chi Q \cos ^{2} \theta\left(\vartheta_{T}-1\right)=0 .
$$


For $Q=0$, equation (A4) reduces to equation (27). In addition, for the special case $\cos ^{2} \theta=0$ equation (A4) reduces to equation (27) with the substitutions $\vartheta_{T} \rightarrow Q+\vartheta_{T}$ and $\vartheta_{s} \rightarrow Q+\vartheta_{s}$. Therefore, for $Q \geq 1$ and $\cos ^{2} \theta=0$, the medium is stabilized for any $\chi, \vartheta_{T}$, and $\vartheta_{s}$. As in Chandrasekhar (1961), I find that for all $\cos ^{2} \theta \neq 0$, if $\vartheta_{T}<1$, the medium is unstable.

\section{APPENDIX B}

\section{MORE GENERAL TREATMENTS OF RADIATION TRANSPORT}

The prescription for radiation transport in $\S 2$ makes several approximations. In particular, it neglects the time dependence of the radiation field and it assumes perfect radiative equilibrium so that the radiation and gas temperatures are identical. The latter assumption is particularly suspect when diffusion is rapid on a scale $k^{-1}$, since radiative equilibrium may not be possible to maintain. In fact, contrary to the results of $\S 2$, when the radiation and gas temperatures are distinguished, the gas acoustic speed in the limit of rapid diffusion should be the adiabatic gas sound speed and not the isothermal gas sound speed (e.g., Mihalas \& Mihalas 1984). More detailed treatments of radiating flows without self-gravity may be found in Dzhalilov et al. (1992), Zhugzhda et al. (1993), and Bogdan et al. (1996). Kaneko \& Morita (2006) provide a detailed treatment of the radiation that distinguishes between scattering and pure absorptive opacity.

For completeness, here I present an analysis similar to $\S 2$, but including the dynamics of the radiation field and allowing for energetic decoupling between the radiation and gas. The set of equations is (cf. eqs. [3]-[7])

$$
\begin{gathered}
\frac{D \rho}{D t}+\rho \nabla \cdot \boldsymbol{v}=0, \\
\frac{D \boldsymbol{v}}{D t}+\frac{1}{\rho} \nabla p_{g}+\nabla \Phi-\kappa \boldsymbol{F} / c=0, \\
\frac{D u_{g}}{D t}+\gamma u_{g} \nabla \cdot \boldsymbol{v}-\kappa \rho c\left(u_{r}-a T^{4}\right)=0, \\
\frac{D u_{r}}{D t}+\frac{4}{3} u_{r} \nabla \cdot \boldsymbol{v}+\nabla \cdot \boldsymbol{F}+\kappa \rho c\left(u_{r}-a T^{4}\right)=0, \\
\nabla^{2} \Phi-4 \pi G \rho=0, \\
\frac{1}{c} \frac{D \boldsymbol{F}}{D t}+\frac{c}{3} \nabla u_{r}+\kappa \rho \boldsymbol{F}=0,
\end{gathered}
$$

where $D / D t=\partial / \partial t+\boldsymbol{v} \cdot \nabla \boldsymbol{v}$ is the Lagrangian derivative, $T$ is the gas temperature, $a=4 \sigma_{\mathrm{SB}} / c$ is the radiation energy density constant, and $\gamma$ is the adiabatic index of the gas. The time derivative of the flux in the Euler equation has been neglected. The perturbation equations are

$$
\begin{gathered}
-i \omega \delta \rho+i \rho \boldsymbol{k} \cdot \delta \boldsymbol{v}=0, \\
-i \omega \delta \boldsymbol{v}+i \boldsymbol{k}\left(\delta p_{g} / \rho\right)+i \boldsymbol{k} \delta \Phi-\kappa \delta \boldsymbol{F} / c=0, \\
-i \omega \delta u_{g}+\gamma u_{g} i \boldsymbol{k} \cdot \delta \boldsymbol{v}+\kappa \rho c\left(\delta u_{r}-A_{g} \delta T\right)=0, \\
-i \omega \delta u_{r}+(4 / 3) u_{r} i \boldsymbol{k} \cdot \delta \boldsymbol{v}+i \boldsymbol{k} \cdot \delta \boldsymbol{F}-\kappa \rho c\left(\delta u_{r}-A_{g} \delta T\right)=0, \\
-k^{2} \delta \Phi-4 \pi G \delta \rho=0, \\
-i \omega \delta \boldsymbol{F}+\left(c^{2} / 3\right) i \boldsymbol{k} \delta u_{r}+(\kappa \rho c) \delta \boldsymbol{F}=0 .
\end{gathered}
$$

where I have taken $\delta\left(a T^{4}\right)=4 a T^{3} \delta T=A_{g} \delta T$ and employed the Jeans swindle. The perturbations to the gas pressure and energy density are written as $\delta p_{g}=c_{T}^{2} \delta \rho+\left.\left(\partial p_{g} / \partial T\right)\right|_{\rho} \delta T$ and $\delta u_{g}=c_{T}^{2} /(\gamma-1) \delta \rho+\left.\left(\partial u_{g} / \partial T\right)\right|_{\rho} \delta T$. Solving equation (B2), the resulting dispersion relation can be written in a number of ways. The form most conducive to comparison with equation (19) is perhaps

$$
\begin{aligned}
\omega^{5}+ & i \Gamma \omega^{4}(2+s)-\omega^{3}\left[\Gamma^{2}(1+s)+\nu^{2}+\gamma c_{T}^{2} k^{2}+\frac{4}{9} \frac{u_{r}}{\rho} k^{2}-4 \pi G \rho\right] \\
& -i \Gamma \omega^{2}\left[\nu^{2} s+\gamma c_{T}^{2} k^{2}\left(\frac{4 s}{3 \gamma}+2\right)+\frac{4}{9} \frac{u_{r}}{\rho} k^{2}(3 \gamma-2+s)-(2+s) 4 \pi G \rho\right] \\
+ & \Gamma^{2} \omega\left[\gamma c_{T}^{2} k^{2}\left(\frac{4 s}{3 \gamma}+1+\frac{\nu^{2}}{\Gamma^{2}}\right)+\frac{4}{9} \frac{u_{r}}{\rho} k^{2}(3 \gamma-3+s)-4 \pi G \rho\left(1+s+\frac{\nu^{2}}{\Gamma^{2}}\right)\right] \\
+ & i \Gamma s \nu^{2}\left(c_{T}^{2} k^{2}-4 \pi G \rho\right)=0,
\end{aligned}
$$

where

$$
s=\frac{A_{g}}{\partial u_{g} /\left.\partial T\right|_{\rho}}=\frac{4 a T^{4}}{u_{g}}, \quad \nu^{2}=\frac{c^{2} k^{2}}{3}, \quad \Gamma=\kappa \rho c, \quad \frac{\nu^{2}}{\Gamma^{2}}=\frac{1}{3} \frac{1}{\left(\kappa \rho k^{-1}\right)^{2}}=\frac{1}{3} \frac{1}{\tau_{k}^{2}}
$$


The latter is the inverse of the optical depth squared across a scale $\sim k^{-1}$. With $G=0$, equation (B3) is identical to equation (101.62) of Mihalas \& Mihalas (1984). ${ }^{9}$ For large optical depth in a radiation pressure-dominated medium, $\nu^{2} / \Gamma^{2} \ll 1 \ll s$ in the third and fifth terms of equation (B3). In addition, for a nonrelativistic medium $\nu^{2} \gg\left(4 u_{r} / 9 \rho\right) k^{2}, c_{T}^{2} k^{2}$, and $4 \pi G \rho$ in the fourth term in equation (B3). Using this ordering, dividing through by the quantity $-\Gamma^{2} s^{2}$, and noting that $\nu^{2} / \Gamma=c k^{2} /(3 \kappa \rho)$ (cf. eqs. [17] and [26]), equation (B3) becomes

$$
-\left(\frac{\omega^{5}}{\Gamma^{2} s^{2}}\right)-\left(\frac{\omega^{4}}{i \Gamma s}\right)+\omega^{3}+i \frac{c k^{2}}{3 \kappa \rho} \omega^{2}-\omega\left(\frac{4}{3} c_{T}^{2} k^{2}+\frac{4}{9} \frac{u_{r}}{\rho} k^{2}-4 \pi G \rho\right)-i \frac{c k^{2}}{3 \kappa \rho}\left(c_{T}^{2} k^{2}-4 \pi G \rho\right) \approx 0 .
$$

This expression should be compared with equation (19). Note that the last four terms in equation (B5) are qualitatively identical to the terms in equation (19) in the radiation pressure-dominated limit $\left(A / C_{V}\right) \approx 1$. The importance of the first and second terms in equation (B5) are measured by the characteristic frequency $\Gamma s$ with respect to the wave frequencies the expression admits. Generically, for sufficiently large $\Gamma s / \omega$, these terms are subdominant. Thus, if these limits hold, the qualitative stability properties outlined in $\S 2$ obtain.

It is simplest to understand the origin of the extra terms in equation (B3) with respect to equation (19) by taking a step back. Neglecting the time dependence of the flux in the last expression in equation (B1), but leaving the rest of the above analysis unchanged, I find that

$$
\begin{aligned}
\omega^{4}+i \Gamma \omega^{3}(1+s+ & \left.\frac{\nu^{2}}{\Gamma^{2}}\right)-\omega^{2}\left(\nu^{2} s+\gamma c_{T}^{2} k^{2}+\frac{4}{9} \frac{u_{r}}{\rho} k^{2}-4 \pi G \rho\right) \\
& -i \Gamma \omega\left[\gamma c_{T}^{2} k^{2}\left(\frac{4 s}{3 \gamma}+1+\frac{\nu^{2}}{\Gamma^{2}}\right)+\frac{4}{9} \frac{u_{r}}{\rho} k^{2}(3 \gamma-3+s)-4 \pi G \rho\left(1+s+\frac{\nu^{2}}{\Gamma^{2}}\right)\right]+s \nu^{2}\left(c_{T}^{2} k^{2}-4 \pi G \rho\right)=0 .
\end{aligned}
$$

The somewhat peculiar terms multiplying $\gamma c_{T}^{2} k^{2}$ and (4/9) $u_{r} k^{2} / \rho$ in the fourth term of the dispersion relation (e.g., $\left.4 s / 3 \gamma\right)$ are made clear by examining the explicit and full expression for the sound speed of the radiation and the gas, at constant total entropy (cf. eq. [18]). When $u_{r} / \rho \gg c_{T}^{2}$, one finds that $c_{s}^{2} \approx(4 / 3) c_{T}^{2}+(4 / 9) u_{r} / \rho$, whereas when $u_{r} / \rho \ll c_{T}^{2}$, one finds that $c_{s}^{2} \approx \gamma c_{T}^{2}+(4 / 3)(\gamma-1) u_{r} / \rho$. This shows that for large and small $s$, these terms reduce to the adiabatic sound speed for the radiation and the gas, respectively. As in deriving equation (B5), if I take $\nu^{2} / \Gamma^{2} \ll 1 \ll s$ (second and fourth terms) and $\nu^{2} \gg\left(4 u_{r} / 9 \rho\right) k^{2}, c_{T}^{2} k^{2}$, and $4 \pi G \rho$ (third term), I find that equation (B6) can be written simply as

$$
-\left(\frac{\omega^{4}}{i \Gamma s}\right)+\omega^{3}+i \frac{c k^{2}}{3 \kappa \rho} \omega^{2}-\omega\left(\frac{4}{3} c_{T}^{2} k^{2}+\frac{4}{9} \frac{u_{r}}{\rho} k^{2}-4 \pi G \rho\right)-i \frac{c k^{2}}{3 \kappa \rho}\left(c_{T}^{2} k^{2}-4 \pi G \rho\right)=0 .
$$

Compare with equation (B5). The importance of $\Gamma s$ is again evident. As emphasized by Bogdan et al. (1996) and Blaes \& Socrates (2003), the characteristic frequency

$$
\omega_{\text {th }}=\Gamma s=\kappa \rho c\left(\frac{4 a T^{4}}{u_{g}}\right)
$$

measures the rate at which energy is exchanged between the matter and the radiation field. When this frequency is large, the energetic coupling is tight and the analysis presented in $\S 2$ is recovered. Thus, for very large $\Gamma s$ and vanishingly small diffusion rate across a scale $k^{-1}-\nu^{2} / \Gamma=c k^{2} /(3 \kappa \rho) \rightarrow 0$ - only the second and third terms in equation (B7) survive: $\omega^{3}-\omega\left(4 c_{T}^{2} k^{2} / 3+(4 / 9) u_{r} k^{2} / \rho-4 \pi G \rho\right) \approx 0$. That is, in the optically thick limit with slow diffusion the radiation pressure contributes to the stability of the system against gravitational collapse. As in $\S 2$, the fact that it appears that the system is stabilized if (4/9) $u_{r} k^{2} / \rho>4 \pi G \rho$, even when (4/3) $c_{T}^{2} k^{2}<4 \pi G \rho$ is an artifact of taking the limit of zero diffusion rate. Taking just the last two terms in equation (B6) (the small $\omega$ limit) and then taking $\nu^{2} / \Gamma^{2} \rightarrow 0$ (high optical depth on a scale $k^{-1}$ ) and then $s \rightarrow \infty$ (for tight energetic coupling between the radiation and the matter, radiation pressure dominated), I find that

$$
\omega \approx-i \frac{\nu^{2}}{\Gamma}\left[\frac{c_{T}^{2} k^{2}-4 \pi G \rho}{(4 / 3) c_{T}^{2} k^{2}+(4 / 9)\left(u_{r} / \rho\right) k^{2}-4 \pi G \rho}\right]=i \frac{c k^{2}}{3 \kappa \rho}\left[\frac{1-\vartheta_{T}}{(4 / 3) \vartheta_{T}+\vartheta_{r}-1}\right]
$$

where

$$
\vartheta_{r}=\frac{4}{9} \frac{u_{r} k^{2}}{\rho} \frac{1}{(4 \pi G \rho)}
$$

is defined in analogy with $\vartheta_{T}$ and $\vartheta_{S}$ (eqs. [22] and [24]). This expression should be compared with equation (32); in the radiation pressuredominated limit they are identical. Thus, as in $\S 2$, I find that even for highly radiation pressure-dominated media, with very large optical depth and strong energetic coupling between matter and radiation, the medium is unstable if $c_{T}^{2} k^{2}<4 \pi G \rho$ (the isothermal Jeans number $\left.\vartheta_{T}<1\right)$. As before, at high- $k$, the characteristic timescale for instability is independent of spatial scale and is simply the Kelvin-Helmholz timescale (cf. eqs. [33] and [37]).

\footnotetext{
${ }^{9}$ Correcting for a sign error in the first term of their eq. (101.58).
} 
Alternatively, taking the limit $\Gamma \rightarrow 0$ in equation (B6), so that $\nu^{2} / \Gamma^{2} \gg s, 1$, and $\nu^{2} s$ equation (B6) becomes

$$
\omega^{3}-\omega\left(\gamma c_{T}^{2} k^{2}-4 \pi G \rho\right) \approx 0 .
$$

Contrary to the discussion of $\S 2$, which showed that in the limit of rapid diffusion the gas acoustic mode speed is the isothermal sound speed $c_{T}$, here I find that when the energetic coupling between the radiation and the gas is weak, acoustic modes propagate at the adiabatic sound speed $\gamma^{1 / 2} c_{T}$, as expected (e.g., Mihalas \& Mihalas 1984). This effect was not accounted for in the analysis of $\S 2$ because perfect energetic coupling was assumed. Equation (B11) shows that in the limit $\Gamma \rightarrow 0$, the classical gas Jeans criterion is obtained and that on scales larger than the Jeans length, the medium is unstable, even if $(4 / 9) u_{r} k^{2} / \rho \gg 4 \pi G \rho$. However, equation (B11) is somewhat deceiving as it may imply to the reader that the medium can be stabilized in the special case $c_{T}^{2} k^{2}<4 \pi G \rho, b u t$ $\gamma c_{T}^{2} k^{2}>4 \pi G \rho$ in the $\Gamma \rightarrow 0$ limit. This is false, and an artifact of having taken $\Gamma=0$ in obtaining equation (B11). Expanding instead to first order in $\Gamma$, I find the unstable mode is

$$
\omega \approx-i \Gamma s\left(\frac{c_{T}^{2} k^{2}-4 \pi G \rho}{\gamma c_{T}^{2} k^{2}-4 \pi G \rho}\right)=i \Gamma s\left(\frac{1-\vartheta_{T}}{\gamma \vartheta_{T}-1}\right),
$$

which shows that in the special case $c_{T}^{2} k^{2}<4 \pi G \rho$, but $\gamma c_{T}^{2} k^{2}>4 \pi G \rho$, the medium is unstable.

Expanding equation (B6) in the high- $k$ limit I find that

$$
\omega \approx \pm \gamma^{1 / 2} c_{T} k-i \frac{3 \Gamma}{2}\left[\left(\frac{4}{9} \frac{u_{r}}{\rho c^{2}}\right)+\frac{s}{3}\left(\frac{\gamma-1}{\gamma}\right)\right],
$$

in agreement with Blaes \& Socrates (2003, their eq. [57]). In a nonrelativistic medium, the second term in square brackets dominates so that $\omega \approx \pm \gamma^{1 / 2} c_{T} k-i \Gamma s(\gamma-1) / 2 \gamma$. Thus, in the high- $k$ limit gas acoustic waves are damped by emission and absorption, again with characteristic damping rate $\sim \Gamma s$. Although in equation (B13) I obtain a wave speed equal to the adiabatic gas sound speed, as in equation (B11), the high- $k$ limit is not identical to the limit $\Gamma \rightarrow 0$ because the gravitational term, which dictates stability/instability, disappears at high $k$. To see this, I write $\omega= \pm\left(\gamma c_{T}^{2} k^{2}-4 \pi G \rho\right)^{1 / 2}+i q$ in equation (B6), take only linear terms in $q$, and then expand to first order as $\Gamma \rightarrow 0$. I find that

$$
\omega \approx \pm\left(\gamma c_{T}^{2} k^{2}-4 \pi G \rho\right)^{1 / 2}-i \frac{3 \Gamma}{2}\left[\left(\frac{4}{9} \frac{u_{r}}{\rho c^{2}}\right)+\frac{s}{3} \frac{(\gamma-1) c_{T}^{2} k^{2}}{\gamma c_{T}^{2} k^{2}-4 \pi G \rho}\right]
$$

For large $k$, equation (B14) reduces to equation (B13). However, on scales where gravity is important, equation (B14) shows that if $\gamma c_{T}^{2} k^{2} \gtrsim 4 \pi G \rho$ then the damping rate of gravity-modified adiabatic gas acoustic waves is altered from the prediction of equation (B13). More importantly, we see explicitly that if $\gamma c_{T}^{2} k^{2}<4 \pi G \rho$ the acoustic mode is unstable.

Taken together, equations (B12) and (B14) show that in the limit of small $\Gamma s$, (1) if both $\vartheta_{T}<1$ and $\gamma \vartheta_{T}<1$, then the medium is unstable, and (2) that if $\vartheta_{T}<1$ and $\gamma \vartheta_{T}>1$, then the medium is also unstable. In addition, equation (B9) shows that when $\Gamma s$ is very large and the energetic coupling between matter and radiation is tight, the medium is also unstable for $\vartheta_{T}<1$, even if $\vartheta_{r} \gg 1$, as in $\S 2$. Thus, one expects that the medium is only globally stable on a scale $k^{-1}$ if the classical Jeans criterion is satisfied and the isothermal Jeans number is larger than unity, $\vartheta_{T}>1$.

\section{B1. FULL SOLUTION TO THE DISPERSION RELATION}

In analogy with $\vartheta_{T}, \vartheta_{s}, \chi$, and $\vartheta_{r}$ (eqs. [22]-[26] and [B10]), I define the quantities

$$
\alpha=\frac{c^{2} k^{2}}{3} \frac{1}{(4 \pi G \rho)}=\frac{\nu^{2}}{(4 \pi G \rho)}, \quad \zeta=\frac{\Gamma s}{(4 \pi G \rho)^{1 / 2}},
$$

where $\zeta$ measures the rate of thermal coupling between radiation and gas in units of the dynamical timescale. With these definitions, the approximate solutions and limits of the previous subsection are illustrated in Figure 3, which presents the full solution to equation (B6) over a broad range of $\nu / \Gamma \propto \tau_{k}^{-1}$ for the parameters $\alpha=10^{7}, \gamma=5 / 3, \vartheta_{r}=3, \vartheta_{T}=1 / 5$ (top left), $\vartheta_{T}=1 / 2$ (top right), $\vartheta_{T}=4 / 5$ (bottom left), and $\vartheta_{T}=3 / 2$ (bottom right) at fixed scale $k^{-1}$. In each panel, I take $s=9(\gamma-1) \vartheta_{r} / \vartheta_{T}$ so that $s=180,36,22.5$, and 12 from left to right, top to bottom, respectively. As in Figures 1 and 2, open and filled circles show the real and imaginary components of $\xi=\omega /(4 \pi G \rho)^{1 / 2}$. Positive complex components indicate unstable modes. Note that in each panel the damping rates $\xi \approx-i \chi$ and $\xi \approx-i \zeta$ are off-scale at intermediate values of $\nu / \Gamma$.

In each panel, because $\vartheta_{r}$-and, by extension, $\vartheta_{s}$-is larger than unity, in the limit of large $\tau_{k}$ (large $\Gamma$, small $\nu / \Gamma$ ) solutions qualitatively identical to those obtained in Figures 1 and 2 are recovered. Thus, the left-hand portions of all panels are similar to Figure 1. The qualitatively new feature of this figure is the small- $\tau_{k}$ regions in each panel. In the top two panels both $c_{T}^{2} k^{2}$ and $\gamma c_{T}^{2} k^{2}$ are less than $4 \pi G \rho\left(\vartheta_{T}<1\right.$ and $\left.\gamma \vartheta_{T}<1\right)$ so that the medium is unstable for any $\nu / \Gamma$. For sufficiently small $\nu / \Gamma$, the growth rate is subdynamical and 

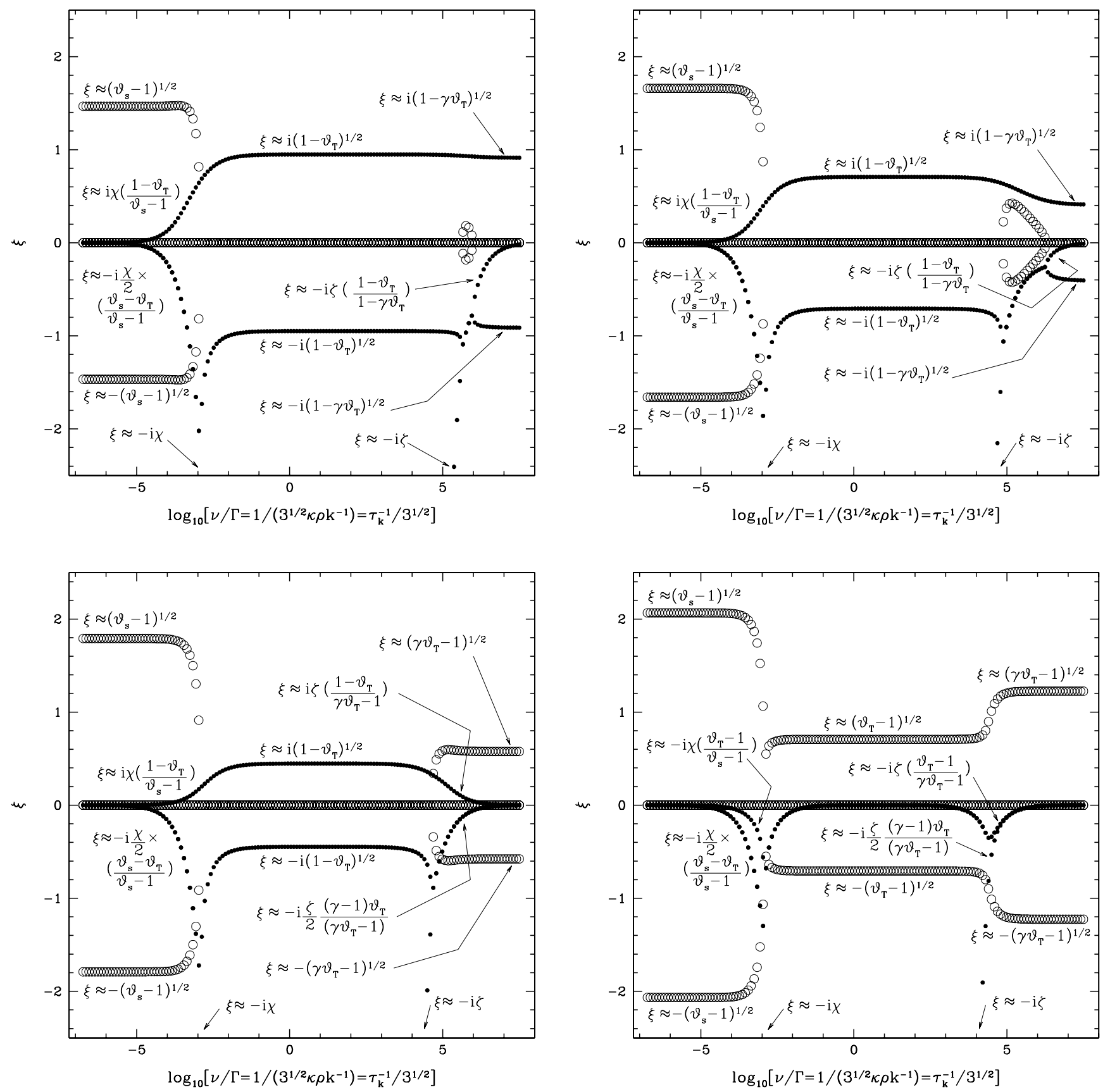

Fig. 3.-Solution to eq. (B6) for $\alpha=10^{7}, \gamma=5 / 3, \vartheta_{r}=3$, and $\vartheta_{T}=1 / 10$ (top left), and for $\vartheta_{T}=1 / 2$ (top right), $\vartheta_{T}=4 / 5$ (bottom left), $\vartheta_{T}=3 / 2$ (bottom right), for a very wide range of $\nu / \Gamma=\tau_{k}^{-1} / 3^{1 / 2}$ at fixed scale $k^{-1}$. Open and filled circles show the real and imaginary parts of the roots $\xi$, respectively. Positive complex roots indicate instability. Components of the dispersion relation are labeled for clarity. The left portion of each panel (large $\tau_{k}$, strong thermal coupling) is qualitatively similar to Figs. 1 and 2. In each panel $\vartheta_{r}>1$ so that $\vartheta_{s}>1$. The bottom left panel shows the special case $\vartheta_{T}<1$ and $\gamma \vartheta_{T}>1$. At very large $\nu / \Gamma$, the gravity- and radiationmodified adiabatic gas only acoustic wave exists and in this region the growth rate for the Jeans instability is suppressed.

equal to the inverse of the Kelvin-Helmholz timescale (cf. eqs. [33] and [37]). Here, the diffusive instability operates. However, for intermediate values of the $\nu / \Gamma$, the growth rate is dynamical $\left[\xi \approx \pm i\left(1-\vartheta_{T}\right)^{1 / 2}\right]$, whereas for very large $\nu / \Gamma, \xi \approx \pm i\left(1-\gamma \vartheta_{T}\right)^{1 / 2}$. In these cases, the medium is classically Jeans unstable.

The bottom left panel is different. Here, $\vartheta_{T}<1$, but $\gamma \vartheta_{T}>1$ (see eq. [B12]). In this special case, two sets of gravity- and diffusionmodified acoustic waves exist: (1) at small $\nu / \Gamma$, the adiabatic radiation pressure dominated acoustic waves are evident (as in the top two panels) and (2) at large $\nu / \Gamma$, adiabatic gas (only) acoustic waves are also present. Note that in this regime (small thermal coupling, small $\tau_{k}$ ) the medium is still unstable, but the growth rate for instability is $\approx \zeta\left(1-\vartheta_{T}\right)\left(\gamma \vartheta_{T}-1\right)^{-1}$ (eq. [B12]) in the special case where both the numerator and the denominator are positive.

The bottom right panel shows a case analogous to the right panels of Figures 1 and 2 with $\vartheta_{T}>1$. Here, the Jeans instability is stabilized at all $\tau_{k}$. At intermediate $\nu / \Gamma, \xi \approx \pm\left(\vartheta_{T}-1\right)^{1 / 2}$, whereas for large values of $\nu / \Gamma, \xi \approx \pm\left(\gamma \vartheta_{T}-1\right)^{1 / 2}$. As in the other panels, in the limit of strong thermal coupling between the radiation and the gas (large $\tau_{k}$, small $\left.\nu / \Gamma\right), \xi \approx \pm\left(\vartheta_{s}-1\right)^{1 / 2}$. 


\section{B2. SCALINGS AND APPLICATIONS}

The qualitative differences at small optical depth or poor thermal coupling between the radiation and the matter in the right-hand portion of each of the panels in Figure 3 with respect to Figures 1 and 2 do not change the conclusions about most of the astrophysical applications discussed in $\S 3$ because fiducial estimates for $\zeta$ and $\alpha$ are very large:

$$
\begin{aligned}
\zeta=\frac{\Gamma s}{(4 \pi G \rho)^{1 / 2}} & =\frac{\kappa \rho c}{(4 \pi G \rho)^{1 / 2}}\left(\frac{4 a T^{4}}{u_{g}}\right) \approx 10^{8} \kappa_{2.5} n_{4}^{-1 / 2} T_{2}^{3} \approx 10^{9} \kappa_{2.5} n_{8}^{-1 / 2} T_{3}^{3} \\
& =\frac{c}{l_{\mathrm{mfp}}} \frac{12(\gamma-1)}{(4 \pi G \rho)^{1 / 2}}\left(\frac{p_{\mathrm{cr}}}{p_{g}}\right) \approx 7 \times 10^{8} l_{0.1 \mathrm{pc}}^{-1} n^{-1 / 2}\left(\frac{p_{\mathrm{cr}}}{p_{g}}\right), \\
\alpha=\frac{\nu^{2}}{4 \pi G \rho} & =\frac{c^{2} k^{2} / 3}{4 \pi G \rho} \approx \frac{c^{2} \pi}{G \rho \lambda^{2}} \approx 7 \times 10^{6} \lambda_{2}^{-2} n_{4}^{-1} \approx 7 \times 10^{8} \lambda_{-1}^{-2} n_{8}^{-1} \\
& \approx 7 \times 10^{8} \lambda_{\mathrm{kpc}}^{-2} n^{-1} .
\end{aligned}
$$

In addition, the ratio $\nu / \Gamma$ is

$$
\begin{aligned}
\frac{\nu}{\Gamma}=\frac{1}{3^{1 / 2}}\left(\frac{k}{\kappa \rho}\right) & =\frac{1}{3^{1 / 2}}\left(\frac{2 \pi}{\kappa \rho \lambda}\right) \approx 0.3 \kappa_{2.5} n_{4}^{-1} \lambda_{2}^{-1} \approx 0.03 \kappa_{2.5} n_{8}^{-1} \lambda_{-1}^{-1} \\
& =\frac{1}{3^{1 / 2}}\left(\frac{2 \pi l_{\mathrm{mfp}}}{\lambda}\right) \approx 4 \times 10^{-4} l_{0.1 \mathrm{pc}} \lambda_{\mathrm{kpc}}^{-1} .
\end{aligned}
$$

The first line of each equation shows the scalings for starbursts and AGN disks, while the second line shows the scaling appropriate for cosmic rays (cf. eq. [46]). For all cases considered, $\zeta$ is very large and $\nu / \Gamma$ is less than unity. The stability properties of these media are thus best represented by the left-hand portion of each of the panels in Figure 3; the radiation and the matter are tightly energetically coupled.

Agol, E., \& Krolik, J. 1998, ApJ, 507, 304

Arons, J. 1992, ApJ, 388, 561

Binney, J., \& Tremaine, S. 1987, Galactic Dynamics (Princeton: Princeton Univ.

Press)

Bisnovatyi-Kogan, G. S., \& Blinnikov, S. I. 1978, Astrophysics, 14, 316 . 1979, Astrophysics, 15, 99

Blaes, O., \& Socrates, A. 2001, ApJ, 553, 987

2003, ApJ, 596, 509

Bogdan, T. J., Knoelker, M., MacGregor, K. B., \& Kim, E.-J. 1996, ApJ, 456, 879

Boulares, A., \& Cox, D. P. 1990, ApJ, 365, 544

Chandrasekhar, S. 1954, ApJ, 119, 7

. 1961, Hydrodynamic and Hydromagnetic Stability (Oxford: Clarendon)

Chang, P., Quataert, E., \& Murray, N. 2007, ApJ, 662, 94

Condon, J. J. 1992, ARA\&A, 30, 575

Condon, J. J., Huang, Z.-P., Yin, Q. F., \& Thuan, T. X. 1991, ApJ, 378, 65

Connell, J. J. 1998, ApJ, 501, L59

Dodelson, S. 2003, Modern Cosmology (Amsterdam: Academic Press)

Downes, D., \& Solomon, P. M. 1998, ApJ, 507, 615

Dzhalilov, N. S., Zhugzhda, Y. D., \& Staude, J. 1992, A\&A, 257, 359

Engelmann, J. J., Ferrando, P., Soutoul, A., Goret, P., \& Juliusson, E. 1990, A\&A, 233, 96

Field, G. B. 1971, ApJ, 165, 29

Gammie, C. F. 1998, MNRAS, 297, 929

Garcia-Munoz, M., Mason, G. M., \& Simpson, J. A. 1977, ApJ, 217, 859

Goldreich, P., \& Lynden-Bell, D. 1965, MNRAS, 130, 97

Goodman, J. 2003, MNRAS, 339, 937

Gorti, U., \& Hollenbach, D. 2004, ApJ, 613, 424

Guo, F., \& Oh, S. P. 2008, MNRAS, 384, 251

Hansen, C. J. 1978, ARA\&A, 16, 15

Hu, W., \& Sugiyama, N. 1996, ApJ, 471, 542

Jeans, J. H. 1928, Astronomy and Cosmogony (Cambridge: Cambridge Univ. Press)

Kaneko, N., \& Morita, K. 2006, Ap\&SS, 305, 349

Kaneko, N., Morita, K., Satoh, T., Toyama, K., Nishimura, M., \& Maekawa, M. 2000, Ap\&SS, 274, 601

Kaneko, N., Tamazawa, S., \& Ono, Y. 1976, Ap\&SS, 42, 441

Kennicutt, R. C., Jr. 1998, ApJ, 498, 541

\section{REFERENCES}

Klessen, R. S., Heitsch, F., \& Mac Low, M.-M. 2000, ApJ, 535, 887

Krolik, J. H. 2007, ApJ, 661, 52

Krumholz, M. R., \& McKee, C. F. 2005, ApJ, 630, 250

Kuwabara, T., \& Ko, C.-M. 2004, J. Korean Astron. Soc., 37, 601

Ledoux, P. 1951, Ann. d'Astrophys., 14, 438

Lithwick, Y., \& Goldreich, P. 2001, ApJ, 562, 279

Lynden-Bell, D. 1966, Observatory, 86, 57

Mac Low, M.-M., \& Klessen, R. S. 2004, Rev. Mod. Phys., 76, 125

Mestel, L. 1965, QJRAS, 6, 161

Mihalas, D., \& Mihalas, B. W. 1983, ApJ, 273, 355

1984, Foundations of Radiation Hydrodynamics (New York: Oxford Univ. Press)

Peebles, P. J. E., \& Yu, J. T. 1970, ApJ, 162, 815

Pier, E. A., \& Krolik, J. H. 1992, ApJ, 399, L23

Scoville, N. 2003, J. Korean Astron. Soc., 36, 167

Scoville, N. Z., Polletta, M., Ewald, S., Stolovy, S. R., Thompson, R., \& Rieke, M. 2001, AJ, 122, 3017

Semenov, D., Henning, T., Helling, C., Ilgner, M., \& Sedlmayr, E. 2003, A\&A, 410,611

Silk, J. 1967, Nature, 215, 1155

1968, ApJ, 151, 459

Sirko, E., \& Goodman, J. 2003, MNRAS, 341, 501

Socrates, A., Blaes, O., Hungerford, A., \& Fryer, C. L. 2005, ApJ, 632, 531

Socrates, A., Davis, S. W., \& Ramirez-Ruiz, E. 2008, ApJ, in press (astro-ph/ 0609796)

Spiegel, E. A. 1957, ApJ, 126, 202

Thompson, T. A., Quataert, E., \& Murray, N. 2005, ApJ, 630, 167 (TQM05)

Thompson, T. A., Quataert, E., Waxman, E., Murray, N., \& Martin, C. L. 2006, ApJ, 645, 186

Turner, J. L., Beck, S. C., \& Ho, P. T. P. 2000, ApJ, 532, L109

Turner, N. J., Blaes, O. M., Socrates, A., Begelman, M. C., \& Davis, S. W. 2005, ApJ, 624, 267

Vranjes, J. 1990, Ap\&SS, 173, 293

Vranjes, J., \& Cadez, V. 1990, Ap\&SS, 164, 329

Weinberg, S. 1971, ApJ, 168, 175

Zhugzhda, Y. D., Dzhalilov, N. S., \& Staude, J. 1993, A\&A, 278, L9 